\title{
A PROBABILISTIC INTERPRETATION OF MARKET SHARES
}

\author{
Apostolos Gerasoulis and Andy S. Kydes
}

December 1979

ENERGY DATA AND MODELS GROUP

NATIONAL CENTER FOR THE ANALYSIS OF ENERGY SYSTEMS

DEPARTMENT OF ENERGY AND ENVIRONMENT

BROOKHAVEN NATIONAL LABORATORY ASSOCIATED UNIVERSITIES, INC.

UNDER CONTRACT NO. EY-76-C-02-0016 WTH THE

UNITED STATES DEPARTMENT OF ENERGY 


\section{DISCLAIMER}

This report was prepared as an account of work sponsored by an agency of the United States Government. Neither the United States Government nor any agency Thereof, nor any of their employees, makes any warranty, express or implied, or assumes any legal liability or responsibility for the accuracy, completeness, or usefulness of any information, apparatus, product, or process disclosed, or represents that its use would not infringe privately owned rights. Reference herein to any specific commercial product, process, or service by trade name, trademark, manufacturer, or otherwise does not necessarily constitute or imply its endorsement, recommendation, or favoring by the United States Government or any agency thereof. The views and opinions of authors expressed herein do not necessarily state or reflect those of the United States Government or any agency thereof. 


\section{DISCLAIMER}

Portions of this document may be illegible in electronic image products. Images are produced from the best available original document. 


\title{
A PROBABILISTIC INTERPRETATION OF MARKET SHARES
}

\author{
Apostolos Gerasoulis and ANDY S. Kydes
}

December 1979

\author{
ENERGY DATA AND MODELS GROUP \\ NATIONAL CENTER FOR THE ANALYSIS OF ENERGY SYSTEMS \\ DEPARTMENT OF ENERGY AND ENVIRONMENT
}

BROOKHAVEN NATIONAL LABORATORY

UPTON, NEW YORK 11973 


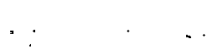

,

\section{DISCLAIMER}

Thic book wạs prepared as an açcount of work sponsorcd by an agency of the Unilieal States Government. Neither the United States Govermuent nor any agency thèreof, nor any of their employees, makes any warranty, express or implied, or assumes any legal liability or responsibility for the accuracy, completentess, ur usefulitess of any' information, apparatus, product, or process disclosed, or represents that its use would not infringe privately nwned rights. Reference herein to any specific commercial prod. uct, process, or service by trade name, trademark, manufacturer, or otherwise, does

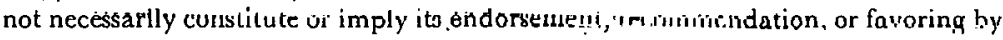
the United States Government or any agency thereof. The views and opiniens of authors expressed herein do not necessarily state or reflect those of the United States Government or any agency thereof.

$$
\text { . }
$$

Printed in the United States of America

$$
\text { Available from }
$$

National Technical Information Service

U.S. Department of Commerce

5285 Port Royal Road

Springfield, VA 22161

Price: Printed Copy Microfiche $\$ 3$.

$$
6-2
$$


The focus of this paper is the development of a steadystate (long-term) characterization of the market allocation process under uncertainty in prices of delivered products. The generic method has been developed with a view toward incorporation of the methodology within large-scale energy models such as the LEAP model of the U.S.DOE or the TESOM model of Brookhaven National Laboratory.

\section{ACKNOWLEDGMENTS}

The authors gratefully acknowledge the superb typing and editorial assistance provided by Phyllis Wolosin, without whose efforts this document could not have been completed, and to M. Dienes for editorial assistance in shaping the final form of this document. 
THIS PAGE

\section{WAS INTENTIONALLY \\ LEFT BLANK}




\section{CONTENTS}

Abstract. . . . . . . . . . . . . . . . . . . . . . . . . . . . . .

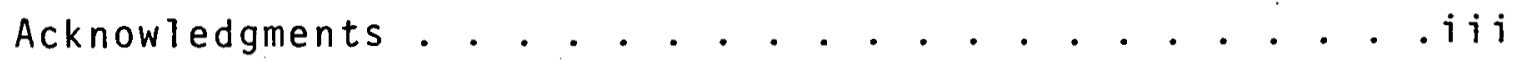

I. Introduction. . . . . . . . . . . . . . . . . 1

II. Mathematical Preliminaries. . . . . . . . . . . 4

III. Steady State Market Shares. . . . . . . . . . . . . 8

The Case for Three Plroducts. . . . . . . . . 9

The N Product Case. . . . . . . . . . 14

IV. Market Shares in the Case of Normal

Distribution Functions. . . . . . . . . . . 18

V. Average Prices. . . . . . . . . . . . . . 27

VI. Sensitivity of Market Shares to the Form of
Probability Function. . . . . . . . . . . . . 32

Uniform Distribution . . . . . . . . . 32

Gamma Distribution.......... . . 35

Weibull Distribution . . . . . . . . . . 36

Additional Examples. . . . . . . . . . . . 40

References. . . . . . . . . . . . . . . . . . . 42 
Figures

1. Probability Density Functions

Page

Three Product Case....................... 9

2. Two Product Case; Uniform Distributions.......... 33

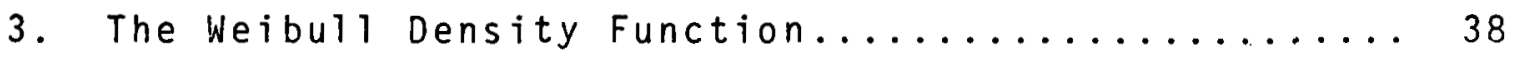

4. Sensitivity of Market Shares for the Weibull

Distribution with Large $\gamma \ldots \ldots \ldots \ldots \ldots \ldots \ldots \ldots . \ldots \ldots$

5. Behavior of the Gamma Distribution............ 40 


\section{INTRODUCTION}

Market penetration from a general perspective is the gradual substitution over time of a new commodity for one or more existing commodities in satisfying basic consumption or production needs. The common measure of penetration is the fraction of the available market serviced by the challenging commodity (i.e., its market share) for each time point considered.

Traditional analyses of market penetration models have focused on the replacement of existing processes by technological innovations which usually reduce unit costs by increased efficiency of utilization or the utilization of less expensive materials. Hurter and Rubenstein ${ }^{1}$ provide a recent extensive review of market penetration literature while Condap and Kydes ${ }^{2}$ have related the Decision Focus, Inc. (DFI) market. allocation representation to past work. Most of the traditional market penetration approaches emphasize either behavioral factors $\left(\mathrm{Bass}^{3}\right.$ ) or economic and technologic factors (Blackman, ${ }^{4-6}$ Mansfield, ${ }^{7}$ Fisher and Pry, ${ }^{8}$ and Peterka ${ }^{9}$ ). $\quad$ Few have introduced the concept of uncertainty in delivered prices. Variations in the prices consumers face can be due to differences in transportation or delivery costs or to market imperfections or institutional barriers. These variations can be substantial, as in the delivered price of No. 2 home heating oil, which varied in 1979 from $\$ 0.60$ per gallon in oil refining states such as New Jersey to well over $\$ 2.00$ per galion in parts of Alaska where it has to be 
flown in. The differences here are primarily due to transportation cost differences. Further, an accounting framework must be established which reconciles the existence of apparently cost ineffective technologies such as solar heating.

Two recent exceptions to the standard approaches include the Industrial Sector Technology Use Model (ISTUM) ${ }^{10}$ developed by Energy and Environmental Associates and Peterka and Fleck's llivel. 11 ISTUM examines the diffusion (penetration) of industrial technologies usina a probabilistic approach. The cost of a technology is derived from the costs of the components (building blocks) of that technology, each of which is defined as an exponential probability distribution. The choice of exponential distributions was motivated in part by the ease of integration of the resulting expression for market shares. The penetration is tempered by behavioral lag adjustments in market penetration, growth in industrial demand, and retirement of old vintage stocks. The choice of exponential frequency distribution is appropriate for 1 ife-death processes and issues of reliability but appears inappropriate for characterizing cost frequency distributions since variations in transportation costs induce peaking at some non-zero value. The normal, gamma or Weibull distributions appear more appropriate.

Peterka and Fleck examine the dynamics of substitution via a logistic substitution model. Fleck views the diffusion of a technology as a social learning process under constraints and uses a stochastic approach to define the diffusion process. 
The weak point of the theory is that the critical parameters have to be measured after the process has begun and are not reducible to other measurements which could be made before the penetration is initiated.

Peterka follows a more classical route taking economics as a driving force. He assumes that an industry has to expand to generate profits. The substitution is driven by differential growth rates, which are driven by differential profits. Although the treatment of Peterka is general and incorporates a Bayesian statistical approach, it contains one potential weakness in that the differential profits are assumed to be constant over long periods of time in order to induce well-behaved logistics.

Our focus in this paper has been to develop the steady-state (long-term) characterization of the market allocation process under uncertainty, assuming that prices are independent random variables as a first order approximation. We derive the resulting market shares formulation for the general $N$ product case where prices are assumed normally distributed. We then derive similar results for the two product case for uniform, gamma and Weibull distributions and have provided several test cases to illustrate the sensitivity of market shares to the choice of distribution type. 


\section{I. MATHEMATICAL PRELIMINARIES}

Useful definitions and identities are summarized here to facilitate the descriptions and derivations of the probabilistic formulation to market shares in the following sections.

Definition 2.1. If $X$ is a continuous random variable then the cumulative (probability) distribution function $F(x)$ is defined by the relation

$$
F(x)=\operatorname{Pr}\{x \leq x ; x \text { is real valued }\} \text {. }
$$

In other words, this is the probability that the random variable $x$ is less than $x$.

Definition 2.2. If $X$ is a continuous random variable and $F(x)$ is the cumulative density function, the probability density function (p.d.f.) of $x, p(x)$, is defined by the relation

$$
F(x)=\int_{-\infty}^{x} p(u) d u \text { for all real } x .
$$

Definition 2.3. If $X$ is a continuous random variable with p.d.f. given by $p(x)$ then the expected value of $x$ is given by

$$
E(x)=\int_{-\infty}^{\infty} x p(x) .
$$

Note that the expected value of any continuous function of $x, q(x)$, is given by

$$
E[q(x)]=\int_{-\infty}^{\infty} q(x) p(x) d x .
$$


See Hogg and Craig ${ }^{12}$ for a thorough introductory discussion of the probability and statistics.

Definition 2.4. A characteristic function, $f(t)$ of a cumulative distribution function $F(x)$, with p.d.f. $p(x)$, is defined by the relation

$$
f(t)=\int_{-\infty}^{\infty} \exp (i t x) p(x) d x .
$$

Definition 2.5. The Fourier transform ${ }^{13}$ of a function $g(x)$ is defined by

$$
G(t) \equiv F[g(x) ; t] \equiv \frac{1}{\sqrt{2 \pi}} \int_{-\infty}^{\infty} \exp (i t x) g(x) d x .
$$

Definition 2.6. The inverse of the Fourier transform

$G(t)$ is defined by

$$
g(x) \equiv \frac{1}{\sqrt{2 \pi}} \int_{-\infty}^{\infty} \exp (-i t x) G(t) d t .
$$

Note that $f(t)$ can be redefined, in view of (2.5) and $(2.6)$ as

$$
f(t)=(\sqrt{2 \pi}) F[p(x) ; t] \text {. }
$$

We now provide some useful definitions or facts from the theory of generalized functions. The reader is directed to Snedion 13 for a more detailed discussion of these facts. It should be noted that the word "distribution" has a different meaning for generalized functions here than it does for probability distributions. Generalized functions, defined below, are used interchangeably with "distributions" in generalized function theory. 
Definition 2.7. The space, $L$, of test functions of rapid descent is the space of functions for which each member, $\phi$, of $L$ and $i$ ts derivatives decrease to zero faster than every power of $|x|^{-1}$. For $n=1$, e.g., $\phi(x) \in R^{1}$, this condition can be written in the form

$$
\left|x_{\phi}^{n_{\phi}(k)}(x)\right| \leq c_{m k}
$$

for all non-negative integers $m$ and $k$ and for all values ur $x$. Ir $\psi(x)$ is an $n$ vector valucd function in the set of reals, $R^{n}$, it will be useful to denote the partial derivative by

$$
\frac{\partial^{k_{1}+k_{2}+\ldots+k r}}{\partial x_{1}^{k_{1}} \partial x_{2}^{k_{2}} \ldots \partial x_{n}^{k_{n}}} \phi\left(x_{1}, x_{2}, \ldots, x_{n}\right) \equiv D^{k_{\phi}}(x)
$$

where $k=\left(k_{1}, k_{2}, \ldots, k_{n}\right)$. The equivalent condition to (2.8) in $R^{n}$ is

$$
|x|^{m} \cdot\left|D^{K}{ }_{\phi}(x)\right| \leq C_{m, k} \text {. }
$$

(See Sneddon, ${ }^{13}$ pages 20-22.)

The function $e^{-x^{2}}$, for example, bclongs to the set of functions of rapid descent, $L$. In general, if $f(x)$ belongs to $L$, then $f^{(k)}(x)$ and the Fourier transform $F[f(x), t]$ also belongs to L (see Ref. 13, p.490).

Definition 2.8, If $f(x)$ belongs to $L$ then the Dirac delta function, $\delta(x)$, is defined by

$$
\int_{-\infty}^{\infty} \delta(x) f(x) d x=f(0) \text {. }
$$


The derivatives of the Dirac delta functions are defined in a similar way.

Definition 2.9. If $f$ belongs to $L$ and $\delta^{(k)}(x)$ represents the $k$-th order derivative of the Dirac delta function, then $\delta^{(k)}(x)$ is defined by

$$
\begin{aligned}
\int_{-\infty}^{\infty} \delta(k)(x) f(x) d x & =(-1)^{k} \int_{-\infty}^{\infty} \delta(x) f(k)(x) d x \\
& =(-1)^{k} f^{k}(0) .
\end{aligned}
$$

Definition 2.10. If $f(x)$ belongs to $L$, then two functions $p(x), q(x)$ "are equal in the space of generalized functions" if and oniy if

$$
\int_{-\infty}^{\infty} p(x) f(x) d x=\int_{-\infty}^{\infty} q(x) f(x) d x
$$

for all $f(x)$ which belong to $L$.

The following useful relations can easily be derived.

The parameter $\alpha$ is assumed to be a real scalar.

$$
\begin{aligned}
& F\left[e^{i \alpha x} ; t\right]=\sqrt{2 \pi} \delta(t+\alpha) \\
& \int_{0}^{\infty} \cos (\alpha+t) x d x=\pi \delta(t+\alpha) \\
& \int_{0}^{\infty} \sin (\alpha+t) x d x= \begin{cases}(\alpha+t)^{-1} ; t \neq-\alpha \\
0 \quad & t=-\alpha\end{cases} \\
& \int_{y}^{\infty} \exp (-i t x) d x=\exp (-i t y)[\pi \delta(t)-i / t] .
\end{aligned}
$$

Finally, we give the Poincaré-Bertrand formula (Tricomi, ${ }^{14}$ p. 172) since it will be used in later deriva- 
tions. The function $\phi(t, x)$ is assumed to be Holder continuous:

$\int_{-\infty}^{\infty} \frac{d t}{t-t_{0}} \int_{-\infty}^{\infty} \frac{\phi(t, x)}{x-t} d x=-\pi^{2} \phi\left(t_{0}, t_{0}\right)+\int_{-\infty}^{\infty} d x \int_{-\infty}^{\infty} \frac{\phi(t, x) d x}{\left(t-t_{0}\right)(x-t)}$

III. STEADY STATE MARKET SHARES

We present in this section a general theoretical derivation of equations which describe the static market

shares of multiple competing products in a probabilistic framework. The probabilistic approach is reasonable since it permits the quantification of variations in price due to (i) different regional costs of transportation, (ii) differing regional taxing policy schemes, (iii) imperfect consumer information/behavior, (iv) differing pricing schemes, and ( $v$ ) imperfect substitution potential.

The case we present here represents the steady state market shares or the case where the market can adjust immediately. Lag time can be incorporated into the adjustment process exogenousiy.

Consider a market for which the prices of $N$ products are random variables $\left(x_{1}, x_{2}, \ldots x_{N}\right)$ with probability density functions $f_{1}\left(x_{1}\right), \ldots, f_{N}\left(x_{N}\right)$. If there is any correlation between prices for these $\mathbb{N}$ products then a joint probability density function $f\left(x_{1}, x_{2}, \ldots, x_{2}\right)$ describes the price interrelationships. An important assumption for the derived model of market shares asserts that consumers act rationally by purchasing the cheapest priced good "known to them." 
Another important assumption asserts that, in the steady state, sufficient capital will be invested to build the industry to satisfy the prescribed market shares. These assumptions imply that the market share of the $i-t h$ product, $S_{i}$, is defined by

$$
s_{i}=\int_{-\infty}^{\infty} d x_{i} \underbrace{\infty}_{\substack{\int_{x_{i}}^{j \neq i} \\ x_{i} \text { deleted }}} d x_{j} \ldots \int_{x_{i}}^{\infty} f\left(x_{1}, x_{2}, \ldots, x_{N}\right) d x_{N}
$$

for $i=1,2, \ldots, N$.

\section{The Case for Three Products}

For the three product case, the p.d.f.'s can be viewed as shown below, and

$$
s_{1}=\int_{-\infty}^{\infty} d x_{1} \int_{x_{1}}^{\infty} d x_{2} \int_{x_{1}}^{\infty} f\left(x_{1}, x_{2}, x_{3}\right) d x_{3} .
$$

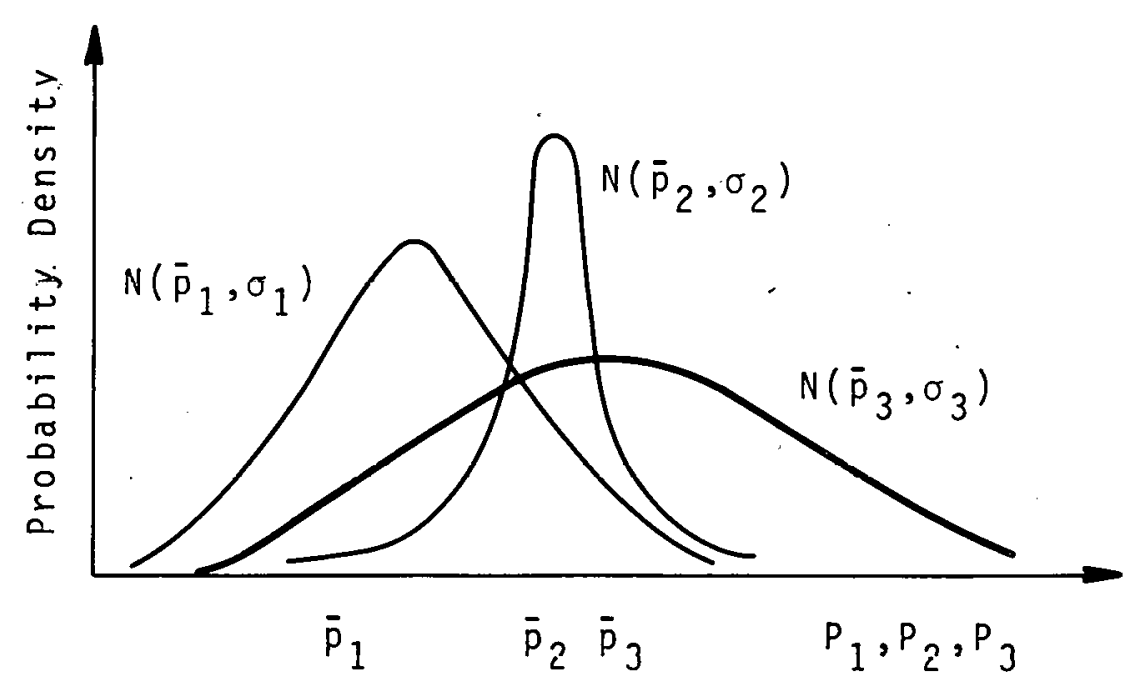

Figure 1. Probability Density Functions Three Product Case 
Assume that $x_{1}, x_{2}, x_{3}$ are independent random, variables;

this ignores the cross price effects and presumes that price is predicated on cost. The fourier transform and its inverse are defined (see Section II) by

$$
F_{j}\left(t_{j}\right)=\frac{1}{\sqrt{2 \pi}} \int_{-\infty}^{\infty} \exp \left(i t_{j} x_{j}\right) f_{j}\left(x_{j}\right) d x_{j}
$$

for $j=1,2,3$;

$$
f_{j}\left(x_{j}\right)=\frac{1}{\sqrt{2 \|}} \int_{-\infty}^{\infty} \exp \left(-i t_{j} x_{j}\right) F_{j}\left(t_{j}\right) d t_{j}
$$

for $j=1,2,3$.

If we introduce $f_{j}\left(x_{j}\right)$, defined in (3.4), into the expression for $s_{1}$ in (3.2) and use the independent random variable assumption we get

$$
\begin{aligned}
s_{1}= & \left(\frac{1}{\sqrt{2 \pi}}\right)^{3} \int_{-\infty}^{\infty} F_{1}\left(t_{1}\right) d t_{1} \int_{-\infty}^{\infty} \exp \left(-i t_{1} x_{1}\right) d x_{1} . \\
& \cdot \int_{-\infty}^{\infty} F_{2}\left(t_{2}\right) d t_{2} \int_{x_{1}}^{\infty} \exp \left(-i t_{2} x_{2}\right) \\
& \cdot \int_{-\infty}^{\infty} F_{3}\left(t_{3}\right) d t_{3} \int_{x_{1}}^{\infty} \exp \left(i t_{3} x_{3}\right) d t_{3} .
\end{aligned}
$$

Substitution of equation (2.16) into (3.5) yields

$$
\begin{gathered}
S_{1}=\left(\frac{1}{\sqrt{2 \pi}}\right)^{3} \int_{-\infty}^{\infty} F_{1}\left(t_{1}\right) d t_{1} \int_{-\infty}^{\infty} \exp \left(-i t_{1} x_{1}\right) d x_{1} \int_{-\infty}^{\infty} F_{2}\left(t_{2}\right) \exp \left(i t_{2} x_{1}\right) \\
\cdot\left(\pi \delta\left(t_{2}\right)-\frac{i}{t_{2}}\right) d t_{2} \cdot I\left(x_{1}\right) \\
I\left(x_{1}\right) \equiv \int_{-\infty}^{\infty} F_{3}\left(t_{3}\right) \exp \left(-i t_{3} x_{1}\right)\left(\pi \delta\left(t_{3}\right)-\frac{i}{t_{3}}\right) d t_{3}
\end{gathered}
$$


Using the identities of

(3.6) yields

$$
\begin{aligned}
s_{1}= & \left(\frac{1}{\sqrt{2 \pi}}\right)^{3} \int_{-\infty}^{\infty} F_{1}\left(t_{1}\right) d t_{1} \int_{-\infty}^{\infty} \exp \left(-i t_{1} x_{1}\right) d x_{1} \int_{-\infty}^{\infty} F_{2}\left(t_{2}\right) \exp \left(-i t_{2} x_{1}\right) \\
& \pi F_{3}(0)\left[\pi \delta\left(t_{2}\right)-\frac{i}{t_{2}}\right] d t_{2} \\
& -\frac{1}{(\sqrt{2 \pi})^{3}} \int_{-\infty}^{\infty} F_{1}\left(t_{1}\right) d t_{1} \int_{-\infty}^{\infty} \exp \left(-i t_{1} x_{1}\right) d t_{1} \int_{-\infty}^{\infty} F_{2}\left(t_{2}\right) \exp \left(-i t_{2} x_{1}\right) \\
& {\left[\pi \delta\left(t_{2}\right)-\frac{i}{t_{2}}\right] d t_{2} \int_{-\infty}^{\infty} \frac{F_{3}\left(t_{3}\right) \exp \left(-i t_{3} x_{1}\right) d t_{3}}{t_{3}} . }
\end{aligned}
$$

On simplifying,

$$
\begin{aligned}
S_{1}= & \left(\frac { 1 } { \sqrt { 2 \pi } } \int ^ { 3 } \left\{\int_{-\infty}^{\infty}\left[F_{1}\left(t_{1}\right) 2 \pi \delta\left(t_{1}\right) \pi^{2} F_{2}(0) F_{3}(0)\right] d t_{1}\right.\right. \\
& -i \pi \sum_{k \neq i, j}^{3} F_{k}(0) \int_{-\infty}^{\infty} F_{1}\left(t_{1}\right) d t_{1} \int_{-\infty}^{\infty} \frac{F_{j}\left(t_{j}\right) d t_{j}}{t_{j}} \int_{-\infty}^{\infty} \exp \left[-i\left(t_{1}+t_{j}\right) x_{1}\right] d x_{1} \\
& -\int_{-\infty}^{\infty} F_{1}\left(t_{1}\right) d t_{1} \int_{-\infty}^{\infty} \exp \left(-i t_{1} x_{1}\right) d x_{1} \int_{-\infty}^{\infty} \frac{d t_{2}}{t_{2}} \int_{-\infty}^{\infty} \frac{F_{3}\left(t_{3}\right) F_{2}\left(t_{2}\right)}{t_{3}} \\
& \left.\exp \left[-i\left(t_{2}+t_{3}\right)\right] d t_{3}\right\} .
\end{aligned}
$$

If $z=t_{2}+t_{3}$ then this becomes

$$
\begin{aligned}
s_{1}= & \left(\frac{1}{\sqrt{2 \pi}}\right)^{3}\left\{2 \pi^{3} F_{1}(0) F_{2}(0) F_{3}(0)-i 2 \pi^{2} F_{3}(0) \int_{-\infty}^{\infty} F_{1}\left(t_{1}\right) d t_{1}\right. \\
& \cdot \int_{-\infty}^{\infty} \frac{F_{2}\left(t_{2}\right)}{t_{2}} \delta\left(t_{1}+t_{2}\right) d t_{2} \\
& -i 2 \pi^{2} F_{2}(0) \int_{-\infty}^{m} F_{1}\left(t_{1}\right) d t_{1} \int_{-\infty}^{m} F_{3}\left(t_{3}\right) \delta\left(t_{1}+t_{3}\right) d t_{3} \\
& -\int_{-\infty}^{\infty} F_{1}\left(t_{1}\right) d t_{1} \int_{-\infty}^{\infty} \exp \left(-i t_{1} x_{1}\right) d t_{1} \int_{-\infty}^{\infty} \frac{d t_{2}}{t_{2}} \int_{-\infty}^{\infty} \frac{F_{3}\left(z-t_{2}\right) F_{2}\left(t_{2}\right)}{z-t_{2}} \exp \left(-i z x_{1}\right) d z .
\end{aligned}
$$


If we interchange the order of integration in the rast integral by using equation (2.17) we obtain

$$
\begin{aligned}
& S_{1}=\frac{1}{(\sqrt{2 \pi})^{3}}\left\{2 \pi^{3} F_{1}(0) F_{2}(0) F_{3}(0)+i 2 \pi^{2} F_{3}(0) \int_{-\infty}^{\infty} \frac{F_{1}\left(t_{1}\right) F_{2}\left(-t_{1}\right)}{t_{1}} d t_{1}\right. \\
& +i 2 \pi^{2} F_{2}(0) \int_{-\infty}^{\infty} \frac{F_{1}\left(t_{1}\right) F_{3}\left(-t_{1}\right)}{t_{1}} d t \\
& -\int_{-\infty}^{\infty} F_{1}\left(t_{1}\right) d t_{1} \int_{-\infty}^{\infty} \exp \left(-i t_{1} x_{1}\right) d x_{1}\left(-\pi^{2} F_{3}(0) F_{2}(0)\right. \\
& \left.\left.1 \int_{-\infty}^{\infty} \exp \left(-i z x_{1}\right) d z \int_{-\infty}^{\infty} \frac{F_{3}\left(z-t_{2}\right) F_{2}}{t_{2}\left(z-t_{2}\right)^{2}} d^{2} d t_{2}\right)\right\} \\
& =\frac{1}{(\sqrt{2 \pi})^{3}}\left\{2 \pi^{3} F_{1}(0) F_{2}(0) F_{3}(0)+i 2 \pi^{2} F_{3}(0) \int_{-\infty}^{\infty} \frac{F_{1}\left(t_{1}\right) F_{2}\left(-t_{1}\right)}{t_{1}} d t\right. \\
& +i 2 \pi^{2} F_{2}(0) \int_{-\infty}^{\infty} \frac{F_{1}\left(t_{1}\right) F_{3}^{0}}{t_{1}} \frac{\left(-t_{1}\right)}{d t} \\
& +2 \pi^{3} F_{1}(0) F_{2}(0) F_{3}(0)-\int_{-\infty}^{\infty} F_{1}\left(t_{1}\right) d t_{1} \\
& \left.-\int_{-\infty}^{\omega}\left(\int_{-\infty}^{\omega} \frac{F_{3}\left(z-t_{2}\right) F_{2}\left(t_{2}\right)}{t_{2}\left(z-t_{2}\right)} d t_{2}\right)\left(\int_{-\infty}^{\omega} \exp \left[-i\left(t_{1}+z\right) x_{1}\right] d x_{1}\right) d z\right\} \\
& =\frac{1}{(\sqrt{2 \pi})^{3}}\left\{2 \pi^{3} F_{1}(0) F_{2}(0) F_{3}(0)+i 2 \pi^{2} F_{3}(0) \int_{-\infty}^{\infty} \frac{F_{1}\left(t_{2}\right) F_{2}\left(-t_{1}\right)}{t_{1}} d t_{1}\right. \\
& +i 2 \pi^{2} F_{2}(0) \int_{-\infty}^{\infty} \frac{F_{1}\left(t_{1}\right) F_{3}\left(-t_{1}\right)}{t_{1}} d t_{1} \\
& +2 \pi^{3} F_{1}(0) F_{2}(0) F_{3}(0)-2 \pi \int_{-\infty}^{\infty} F_{1}\left(t_{1}\right) \int_{-\infty}^{\infty} \frac{F_{3}\left(-t_{1}-t_{2}\right) F_{2}\left(t_{2}\right)}{t_{2}\left(-t_{1}-t_{2}\right)} d t_{2} \cdot
\end{aligned}
$$


If we define

$$
I=\int_{\infty}^{\infty} F_{1}\left(t_{1}\right) \int_{\infty}^{\infty} \frac{F_{3}\left(-t_{1}-t_{2}\right) F_{2}\left(t_{2}\right)}{t_{2}\left(-t_{1}-t_{2}\right)} d t_{2}
$$

and make the change of variables, $t_{2}=-z$, then

$$
\begin{aligned}
I & =\int_{-\infty}^{\infty} F_{1}\left(t_{1}\right) d t_{1} \int_{-\infty}^{\infty} \frac{F_{3}\left(-t_{1}+z\right) F_{2}(-z)}{z\left(t_{1}-z\right)} d z \\
& =\int_{-\infty}^{\infty} \frac{d z}{z} \int_{-\infty}^{\infty} \frac{F_{1}\left(t_{1}\right) F_{2}(-z) F_{3}\left(-t_{1}+z\right)}{t_{1}-z} d z+\pi^{2} F_{1}(0) F_{2}(0) F_{3}(0) .
\end{aligned}
$$

Finally if we introduce $I$ into the last equation for $S_{1}$ we obtain the expression

$$
\begin{gathered}
S_{1}=\frac{1}{(\sqrt{2 \pi})^{3}}\left\{2 \pi^{3} F_{1}(0) F_{2}(0) F_{3}(0)+i 2 \pi^{2} \sum_{\substack{k=2,3 \\
j \neq k}} F_{k}(0) \int_{-\infty}^{\infty} \frac{F_{1}\left(t_{1}\right) F_{j}\left(-t_{1}\right)}{t_{1}} d t_{1}\right. \\
\left.-2 \pi \int_{-\infty}^{\infty} \frac{d z}{z} F_{2}(-z) \int_{-\infty}^{\infty} \frac{F_{1}\left(t_{1}\right) F_{3}\left(-t_{1}+z\right)}{t_{1}-z} d t_{1}\right\} .
\end{gathered}
$$

Now we can easily obtain $S_{2}, S_{3}$ by interchanging the index 1 with 2 or 3 in $(3.7)$ :

$$
\begin{aligned}
S_{2}= & \frac{1}{(\sqrt{2 \pi})^{3}}\left\{2 \pi^{3} F_{1}(0) F_{2}(0) F_{3}(0)+i 2 \pi^{2} \sum_{\substack{k=1,3 \\
j \neq k}} F_{k}(0)\right. \\
& \cdot \int_{-\infty}^{\infty} \frac{F_{2}\left(t_{1}\right) F_{j}\left(-t_{1}\right)}{t_{1}} d t_{1}-2 \pi \int_{-\infty}^{\infty} \frac{d z}{z} F_{1}(-z) \\
& \left.\cdot \int_{-\infty}^{\infty} \frac{F_{2}\left(t_{1}\right) F_{3}\left(-t_{1}+z\right)}{t_{1}-z} d t_{1}\right\},
\end{aligned}
$$




$$
\begin{gathered}
S_{3}=\frac{1}{(\sqrt{2 \pi})^{3}}\left\{2 \pi^{3} F_{1}(0) F_{2}(0) F_{3}(0)+.2 \pi^{2} \sum_{k=1,2} F_{k}(0)\right. \\
\cdot \int_{-\infty}^{\infty} \frac{F_{3}\left(t_{1}\right) F_{j}\left(-t_{1}\right)}{t_{1}} d t_{1} \quad \\
\left.-2 \pi \int_{-\infty}^{\infty} \frac{d z}{z} F_{2}(-z) \int_{-\infty}^{\infty} \frac{F_{3}\left(t_{1}\right) F_{1}\left(-t_{1}+z\right)}{t_{1}-z} d t_{1}\right\} .
\end{gathered}
$$

\section{The $\mathbb{N}$ Product Case}

The $N$ product generalization to markct shares can be found by following the samc steps as in the case $N-3$, then

$$
S_{m}=\frac{2}{(\sqrt{2 \pi})} N\left(\pi^{N} \prod_{j=1}^{N} F_{j}(0)+\sum_{k=1}^{N-1} A_{k, m}\right)
$$

where $m=1,2, \ldots, N$, and

$$
\begin{aligned}
& A_{k, m}=(-i)^{k} \pi^{N-k} \overbrace{j \neq m} \sum_{j \neq j+1} \sum_{l=\rho+1} \cdots \sum_{n}^{N}\left(\prod_{\mu \neq j, \rho, l, \ldots, n} F_{\mu}(0)\right. \\
& k \\
& \cdot \int_{-\infty}^{\infty F_{j}\left(t_{j}\right)} \frac{t_{j}}{t_{j}} \int_{-\infty}^{\infty} \frac{F_{\rho}\left(t_{\rho}\right)}{t_{\rho}} d t_{\rho} \int_{-\infty}^{\infty} \frac{F_{l}\left(t_{\ell}\right)}{t_{\ell}} d t_{\ell} \cdots \\
& \cdot \int_{-\infty}^{\infty} \int_{-\infty}^{\infty} \frac{F_{m}\left(-t_{j}-t_{p}-t_{\ell}-\ldots-t_{n}\right) F_{n}\left(t_{n}\right) d t_{n}}{t_{n}}, m=1,2, \ldots, N .
\end{aligned}
$$

Equations (3.10), (3.11) become, for $N=2, m=1$,

$$
\begin{aligned}
& S_{1}=\frac{2}{\sqrt{2 \pi}}\left[\pi^{2} F_{1}(0) F_{2}(0)+A_{11}\right], \\
& A_{11}=-i \pi \int_{-\infty}^{\infty} \frac{F_{1}\left(t_{2}\right) F_{22}\left(t_{2}\right)}{t_{2}} d t_{2}
\end{aligned}
$$


Similarly for $N=3, m=1$ we have

$$
\begin{aligned}
S_{i} & =\frac{2}{(\sqrt{2 \pi})^{3}}\left(\pi^{3} F_{1}(0) F_{2}(0) F_{3}(0)+\sum_{k=1}^{2} A_{k, 1}\right), \\
A_{11} & =(-i) \pi^{2} \sum_{j=2}^{3}\left(\prod_{\mu \neq j} F_{\mu}(0)\right) \int_{-\infty}^{\infty} \frac{F_{1}\left(-t_{j}\right) F_{j}\left(t_{j}\right)}{t_{j}} d t_{j}, \\
A_{12} & =-\pi \sum_{\substack{j=2 \\
j \neq 1}}^{2} \sum_{\rho=3}^{3}\left(\prod_{\mu \neq j, \rho} F_{\mu}(0)\right) \int_{-\infty}^{\infty} \frac{F_{j}\left(t_{j}\right)}{t_{j}} d t_{j} \int_{-\infty}^{\infty} \frac{F_{1}\left(-t_{j}-t_{\rho}\right) F_{\rho}\left(t_{\rho}\right)}{t_{\rho}} d t_{\rho} \\
& =-\pi \int_{-\infty}^{\infty} \frac{F_{2}\left(t_{2}\right)}{t_{2}} d t_{2} \int_{-\infty}^{\infty} \frac{F_{1}\left(-t_{2}-t_{3}\right) F_{3}\left(t_{3}\right)}{t_{3}} d t_{3} .
\end{aligned}
$$

We can easily see that equations (3.12) and (3.13) are exactly the same as equation (3.7).

We conclude this section by showing that the market shares sum

$$
\sum_{i=1}^{N} S_{i}=1 \text {. }
$$

For simplicity we will consider the case $N=3$.

If we add equations (3.7), (3.8), and (3.9) we see that the integrals

$$
\int_{\infty}^{\infty} \frac{F_{i}(t) F_{j}(-t)}{t} d t
$$

cancel and we obtain the equation

$$
\begin{aligned}
S_{1}+S_{2} & +S_{3}=\frac{1}{(\sqrt{2 \pi})^{3}}\left\{3 \cdot 2 \pi^{3} F_{1}(0) F_{2}(0) F_{3}(0)\right. \\
& -z_{11}\left(\int_{-\infty}^{\infty} \frac{d z}{z} F_{2}(-L) \int_{-\infty}^{\infty} \frac{F_{1}\left(t_{1}\right) F_{a}\left(-t_{1}+z\right)}{t_{1}-z} d t_{1}\right.
\end{aligned}
$$




$$
\begin{aligned}
& +\int_{-\infty}^{\infty} \frac{d z}{z} F_{1}(-z) \int_{-\infty}^{\infty} \frac{F_{2}\left(t_{1}\right) F_{3}\left(-t_{1}+z\right)}{t_{1}-z} d t_{1} . \\
& \left.\left.+\int_{-\infty}^{\infty} \frac{d z}{z} F_{2}(-z) \int_{-\infty}^{\infty} \frac{F_{3}\left(t_{1}\right) F_{1}\left(-t_{1}+z\right)}{t_{1}-z} d t_{1}\right)\right\} .
\end{aligned}
$$

Consider the sum

$$
\begin{gathered}
I=\int_{-\infty}^{\infty} \frac{F_{2}(-z)}{z} d z \int_{-\infty}^{\infty} \frac{F_{1}\left(t_{1}\right) F_{3}\left(-t_{1}+z\right)}{t_{1}-z} d t_{1}+\int_{-\infty}^{\infty} \frac{F_{2}(-z)}{z} d z \\
\cdot \int_{-\infty}^{\infty} \frac{F_{3}\left(t_{1}\right) F_{1}\left(-t_{1}+z\right)}{t_{1} z} d t
\end{gathered}
$$

and set $-t_{1}+z=u$ in the first integral in (3.15). Then

$$
\begin{aligned}
I= & -\int_{-\infty}^{\infty} \frac{F_{2}(-z)}{z} d z \int_{-\infty}^{\infty} \frac{F_{1}(-u t z) F_{3}(u)}{u} d u+\int_{-\infty}^{\infty} \frac{F_{2}(-z)}{z} d z \\
& \cdot \int_{-\infty}^{\infty} \frac{F_{3}\left(t_{1}\right) F_{1}\left(-t_{1}+z\right)}{t_{1}-z} d t_{1} \\
= & \int_{-\infty}^{\infty} \frac{F_{2}(-z)}{z} d z \int_{-\infty}^{\infty} F_{1}\left(-t_{1}+z\right) F_{3}\left(t_{1}\right)\left\{\frac{1}{t_{1}-z}-\frac{1}{t_{1}}\right\} d t_{1} .
\end{aligned}
$$

Hence,

$$
I=\int_{-\infty}^{\infty} F_{2}(-z) d z \int_{-\infty}^{\omega_{F_{1}}\left(-t_{1}+z\right) F_{3}\left(t_{1}\right)} \frac{F_{1}\left(t_{1}-z\right)}{t_{1}} d t .
$$

Consider also the integral

$$
\begin{aligned}
I_{1} & =\int_{-\infty}^{\infty} \frac{F_{1}(-z)}{z} d z \int_{-\infty}^{\infty} \frac{F_{2}\left(t_{1}\right) F_{3}\left(-t_{1}+z\right)}{t_{1}-z} d t_{1} \\
& =-\pi^{2} F_{1}(0) F_{2}(0) F_{3}(0)+\int_{-\infty}^{\infty} d t_{1} \int_{-\infty}^{\infty} \frac{F_{1}(-z) F_{1}\left(t_{1}\right) F_{3}\left(-t_{1}+7\right)}{z\left(t_{1}-z\right)} d z \\
& =-\pi^{2} F_{1}(0) F_{2}(0) F_{3}(0)+\int_{-\infty}^{\infty} F_{2}(z) \int_{-\infty}^{\infty} \frac{F_{1}\left(-t_{1}\right) F_{3}\left(-z+t_{1}\right)}{t_{1}\left(z-t_{1}\right)} d t_{1}
\end{aligned}
$$

$-16-$ 
and if we substitute $z$ for $-z$ and $t_{1}$ for $-t_{1}$ in the last integral we find that

$$
I_{1}=-\pi^{2} F_{1}(0) F_{2}(0) F_{3}(0)+\int_{-\infty}^{\infty} F_{2}(-z) d z \int_{-\infty}^{\infty} \frac{F_{1}\left(t_{1}\right) F_{3}\left(z-t_{1}\right)}{t_{1}\left(z-t_{1}\right)} d t t_{1} .
$$

Set $t_{1}=z-u$; then

$$
\begin{aligned}
I_{1} & =-\pi^{2} F_{1}(0) F_{2}(0) F_{3}(0)+\int_{-\infty}^{\infty} F_{2}(-z) d z \int_{-\infty}^{\infty} \frac{F_{1}(z-u) F_{3}(u)}{(z-u) u} d u \\
& =-\pi^{2} F_{1}(0) F_{2}(0) F_{3}(0)-\int_{-\infty}^{\infty} F_{2}(-z) d z \int_{-\infty}^{\infty} \frac{F_{1}(z-u) F_{3}(u)}{u(u-z)} d u .
\end{aligned}
$$

Adding $I$ and $I_{1}$

$$
I_{1}+I=-\pi^{2} F_{1}(0) F_{2}(0) F_{3}(0)
$$

so that (3.14) becomes

$$
\begin{aligned}
S_{1}+S_{2}+S_{3} & =\frac{1}{(\sqrt{2 \pi})^{3}}\left\{3 \cdot 2 \pi^{3} F_{1}(0) F_{2}(0) F_{3}(0)-2 \pi\left(I+I_{1}\right)\right\} \\
& =\frac{1}{(\sqrt{2 \pi})^{3}}\left\{4 \cdot 2 \pi^{3} F_{1}(0) F_{2}(0) F_{3}(0)\right\} .
\end{aligned}
$$

If we use the relation $F_{j}(0)=\frac{1}{\sqrt{2 \pi}}, j=1,2,3$, then

(3.18) becomes

$$
\sum_{j=1}^{3} s_{j}=1
$$




\section{IV: MARKET SHARES IN THE CASE OF NORMAL DISTRIBUTION FUNCTIONS}

In this section we consider the special case of normally distributed price random variables. First, an analytical method for the case of three products $(N=3)$ is studied. The same analytical procedure may be used for the general case of $\mathrm{N}$ products. Finally a direct numerical approximation is developed and the numerical results are compared with the analytical ones for the case $N=2$.

Let

$$
\left.f_{j}\left(x_{j}\right)=\frac{i}{\sqrt{2 \pi} \sigma_{j}} \exp \left[-\left(x_{j}-\bar{p}_{j}\right)^{2} / 2 \sigma_{j}^{2}\right)\right] . \text {. }
$$

From Sneddon ${ }^{13}$ we see that

$$
\begin{aligned}
F_{j}\left(t_{j}\right) & =\frac{1}{\sqrt{2 \pi}} \int_{-\infty}^{\infty} \exp \left(i t_{j} x_{j}\right) f_{j}\left(x_{j}\right) d x_{j} \\
& =\frac{1}{\sqrt{2 \pi}} \exp \left(i t_{j} \bar{p}_{j}\right) \exp \left[-\left(\sigma_{j}^{2} t_{j}^{2}\right) / 2\right] .
\end{aligned}
$$

We write equation (3.7) as

$$
S_{1}=\frac{1}{(\sqrt{2 \pi})^{3}}\left\{2 \pi^{3} F_{1}(0) F_{2}(0) F_{3}(0)+2 \pi^{2} \sum_{\substack{j=2 \\ k \neq j}}^{3} F_{j}(0) I_{k}-2 \cdots I\right\}
$$

where

$$
\begin{aligned}
I_{k} & =i \int_{-\infty}^{\infty} \frac{F_{1}(t) F_{k}(-t)}{t} d t, \\
I & =\int_{-\infty}^{\infty} \frac{F_{2}(-z)}{z} d z \int_{-\infty}^{\infty} \frac{\Gamma_{1}(t) \Gamma_{3}(-t+z)}{t-z} d t .
\end{aligned}
$$

If we introduce (4.2) in the first integral in (4.4), we have $\bar{p}_{k 1}=\bar{p}_{k}-\bar{p}_{1}, \sigma_{1 k}^{2}=\sigma_{1}^{2}+\sigma_{k}^{2}$, and 


$$
\begin{aligned}
I_{k} & =\frac{i}{2 \pi} \int_{-\infty}^{\infty} \frac{\exp \left(-i t \bar{p}_{k l}-\frac{t^{2} \sigma^{2} l k}{2}\right)}{t} d t \\
& =\frac{1}{2 \pi} \int_{-\infty}^{\infty} \frac{\exp \left(-\frac{x^{2}}{2}-\frac{i x \bar{p}_{k l}}{\sigma_{1 k}}\right)}{x} d x
\end{aligned}
$$

where we have made the change of variable, $t=\frac{x}{\sigma_{1 k}}$. If we define

$$
Q(x)=\frac{1}{\sqrt{2 \pi}} \int_{-\infty}^{x} \exp \left(-\frac{t^{2}}{2}\right) d t
$$

then

$$
Q(x)=\frac{1}{2}\left(1+\operatorname{erf}\left(\frac{x}{\sqrt{L}}\right)\right)
$$

where (see ref. 13, p. 155)

$$
\operatorname{erf}(u)=\frac{2}{\sqrt{\pi}} \int_{0}^{u} \exp \left(-t^{2}\right) d t=1-\operatorname{erfc}(u) .
$$

From equation (4.6) we have

$$
\begin{aligned}
Q(x) & =\frac{1}{\sqrt{2 \pi}} \int_{-\infty}^{x} d t \int_{-\infty}^{\infty} \exp \left(-\frac{y^{2}}{2}-i y t\right) d y \\
& =\frac{1}{2 \pi} \int_{-\infty}^{\infty} \exp \left(-\frac{y^{2}}{2}\right) d y \int_{-\infty}^{x} \exp (-i y t) d t .
\end{aligned}
$$

Let $t=-z+x, d t=-d z$. Then

$$
\begin{aligned}
Q(x) & =\frac{1}{2 \pi} \int_{-\infty}^{\infty} \exp \left(-\frac{y^{2}}{2}-i y x\right) d y \int_{0}^{\infty} \exp (i y z) d z \\
& =\frac{1}{2 \pi} \int_{-\infty}^{\infty} \exp \left(-\frac{y^{2}}{2}-i y x\right) d y\left\{\pi \delta(y)+\frac{i}{y}\right\} \\
& =\frac{1}{2}+\frac{i}{2 \pi} \int_{-\infty}^{\infty} \frac{\exp \left(-i y x-\frac{y^{2}}{2}\right)}{y} d y .
\end{aligned}
$$

From $(4.5)$ and $(4.10)$ we find

$$
Q\left(\frac{\bar{p}_{k 1}}{\sigma_{1 k}}\right)-\frac{1}{2}=I_{k} \text {. }
$$


If we use relation (4.7) in (4.11) we find that $I_{k}$ is given by

$$
I_{k}=\frac{1}{2} \operatorname{erf}\left(\frac{\bar{p}_{k\rceil}}{\sqrt{2} \sigma_{1 k}}\right) \text {. }
$$

To evaluate I in (4.4) we use the relation (cf. Erdelyi et al. ${ }^{15}$ )

$$
\begin{aligned}
& \int_{-\infty}^{\infty}(i x)^{n} \exp \left(-a^{2} x^{2}\right) \exp (-i x y) d x=n^{\frac{1}{2} 2} 2^{-n} a^{-n-1} \exp \left(-y^{2} \cdot \frac{a^{-2}}{8}\right) D_{n 1}\left(2^{-\frac{1}{2}} a^{-1} y\right), \\
& D_{n}(z)=(-1)^{n} \exp \left(\frac{z^{2}}{4}\right) \frac{d^{n}}{d z^{n}}\left[\exp \left(\frac{z^{2}}{2}\right)\right], \\
& \operatorname{Re}(a)>0, \\
& n>-1 .
\end{aligned}
$$

Making the change of variables $t_{1}-z=u$ in

(4.4) gives

$$
\begin{aligned}
& I=\int_{-\infty}^{\infty} \frac{F_{2}(-z)}{z} d z \int_{-\infty}^{\infty} \frac{F_{1}(u+z) F_{3}(-u)}{u} d u \\
&=\frac{1}{(\sqrt{2 \pi})^{3}} \int_{-\infty}^{\infty} \frac{\exp \left(-i z \bar{p}_{2}-\frac{\sigma_{2}^{2} z^{2}}{2}\right)}{z} d z \\
& \cdot \int_{-\infty}^{\infty} \frac{\exp \left[i(z+u) \bar{p}_{1}\right] \exp \left(-\frac{\sigma_{1}^{2}(z+u)^{2}}{2}\right) \exp \left(i u \bar{p}_{3}\right) \exp \left(-\frac{\sigma_{3}^{2} u^{2}}{2}\right)}{u} d u \\
& \frac{1}{(\sqrt{2} \bar{\pi})^{3}} \int_{-\infty}^{\infty} \frac{\exp \left(-i z \bar{p}_{21}\right) \exp \left(-\frac{\sigma_{12}^{2} z^{2}}{2}\right)}{z} d z \\
& \cdot \int_{-\infty}^{\infty} \frac{\exp \left(-i \bar{p}_{31} u\right) \exp \left(-\frac{\sigma_{13}^{2} u^{2}}{2}-\sigma_{1}^{2} z u\right)}{u} d u, \\
& \bar{p}_{k \ell}=\bar{p}_{k}-\bar{p}_{\ell}, \\
& \sigma_{k \ell}^{2}=\sigma_{k}^{2}+\sigma_{\ell}^{2} .
\end{aligned}
$$


A Taylor expansion of $\exp \left(-\sigma_{1}^{2} z u\right)$ gives $\exp \left(-\sigma_{1}^{2} z u\right)=1-\sigma_{1}^{2} z u+\frac{\left(\sigma_{1}^{2} z u\right)^{2}}{2 !}+\ldots$ $(-1)^{n} \frac{\left(\sigma_{1}^{2} z u\right)^{n}}{n !}+\ldots$ and introducing it in (4.15) gives

$$
\begin{aligned}
I= & \frac{1}{(\sqrt{2 \pi})^{3}}\left\{\int_{-\infty}^{\infty} \frac{\exp \left(-i z \bar{p}_{21}\right) \exp \left(-\frac{\sigma_{12}^{2} z^{2}}{2}\right)}{z} \cdot d z\right. \\
& \cdot \int_{-\infty}^{\infty} \frac{\exp \left(-i \bar{p}_{31} u-\frac{\sigma_{13}^{2} u^{2}}{2}\right)}{u} d u-\sigma_{1}^{2} \int_{-\infty}^{\infty} \exp \left(-i z \bar{p}_{21}\right) \exp \left(-\frac{\sigma_{12}^{2} z^{2}}{2}\right) d z \\
& \cdot \int_{-\infty}^{\infty} \exp \left(-i u \bar{p}_{31}\right) \exp \left(-\frac{\sigma_{13}^{2} u^{2}}{2}\right) d u+\sigma_{1}^{4} \int_{-\infty}^{\infty} \exp \left(-i z \bar{p}_{21}\right) \exp \left(-\frac{\sigma_{12}^{2} z^{2}}{2}\right) z d z \\
& \left.\cdot \int_{-\infty}^{\infty} \exp \left(-i \bar{p}_{31} u\right) \exp \left(-\frac{\sigma_{13}^{2} u^{2}}{2}\right) u d u-\ldots\right\} .
\end{aligned}
$$

After using $(4.5),(4.13),(4.14)$ we get

$$
I=\frac{1}{(\sqrt{2 \pi})^{3}}\left\{\frac{\pi}{i} \operatorname{erf}\left(\frac{\bar{p}_{21}}{\sqrt{2} \sigma_{12}}\right) \cdot \frac{\pi}{i} \operatorname{erf}\left(\frac{\bar{p}_{31}}{\sigma_{13}}\right) \ldots .\right\} \text {. }
$$

From (4.16), (4.12), and (4.3) we can compute the nominal market share $\mathrm{S}_{1}$.

The previous method of evaluating the market shares can be extended for the general case of $N$ products. It is more convenient, however, to use direct numerical methods for the evaluation of market shares. Since we cañ evaluate the market shares analytically, the numerical results and the efficiency of the numerical method can be tested.

From equations $(3.1),(4.1)$ we have 


$$
\begin{aligned}
& s_{i}=\frac{\prod^{-j} \sigma_{i}}{\sqrt{2 \pi}} \int_{-\infty}^{\infty} \exp \left(-\frac{\left(x_{j}-\bar{p}_{i}\right)^{2}}{2 \sigma_{j}^{2}}\right) d x_{i} \\
& \prod_{\substack{j=1 \\
j \neq i}}\left\{\frac{1}{\sqrt{2 \pi} \sigma_{j}} \int_{x_{i}}^{\infty} \exp \left(-\frac{\left(x_{j}-\bar{p}_{j}\right)^{2}}{2 \sigma_{j}{ }^{2}}\right) d x_{j}\right\}, \\
& i=1,2, \ldots, N .
\end{aligned}
$$

Let us define

$$
E\left(x_{i}\right)=\frac{1}{\sqrt{2 \pi} \sigma_{j}} \int_{x_{i}}^{\infty} \exp \left(-\frac{\left(x_{j}-\bar{p}_{j}\right)^{2}}{2 \sigma_{j}{ }^{2}}\right) d x_{j} .
$$

I hen

$$
E\left(x_{j}\right)=\frac{1}{v^{\prime} \pi} \int_{\frac{x_{j}-p_{j}}{\sqrt{2} \sigma_{j}}}^{\infty} \exp \left(-u^{2}\right) d u=\frac{1}{2} \operatorname{erfc}\left(\frac{x_{j}-\bar{p}_{j}}{\sqrt{2} \sigma_{j}}\right)
$$

where

$$
\operatorname{erfc}(u)=\frac{2}{\sqrt{\pi}} \int_{u}^{\infty} \exp \left(-t^{2}\right) d t
$$

is defined in Sneddon, 13 page 155 .

There are several numerical methods for evaluating $E\left(x_{i}\right)$; see Abramowitz et al. 16 We use a simple numerical method, the trapezoidal rule, for the evaluation of $E\left(x_{j}\right)$ and $S_{i}$. If a function is piecewise continuous then the trapezoidal rule is defined by

$$
\int_{a}^{b} f(x) d x=n\left(\frac{f_{0}}{2}+r_{1}+\ldots \cdot f_{n-1}+\frac{f_{n}}{2}\right),
$$

where

$$
h=\frac{b-a}{n}, \quad t_{j}=a+j h, \quad f_{j}=f\left(t_{j}\right), j=0,1, \ldots, n .
$$


From (4.17) we have

$$
S_{i}=\frac{1}{\sqrt{\pi}} \int_{-\infty}^{\infty} \exp \left(-u^{2}\right) f(u) d u, \quad f(u)=\prod_{\substack{j=1 \\ j \neq i}}^{N} \frac{1}{\sqrt{\pi}} \int_{v}^{\infty} \exp \left(-t^{2}\right) d t,
$$

where $v=\left(u \frac{\sigma_{i}}{\sigma_{j}}+\frac{\bar{p}_{i j}}{\sqrt{2} \sigma_{j}}\right)$.

When the representation of $f(u)$ is exact then the trapezoidal rule yields very accurate results for the approximation of $S_{j}$ over the interval $[-6,6]$ since exp. $\left(-u^{2}\right) f(u)$ has derivatives almost equal to zero for $u \leq-6$ and $u \geq 6$ (cf. Dahlquist et al..,7 p. 296). When $f(u)$ is itself.approximated then the trapezoidal rule will give an approximation of order $0\left(h^{2}\right)$, since $v$ may become zero and the advantage of having small derivatives in $f(u)$ is no longer useful. One can use a higher order accuracy method for better results, e.g., Gaussian quadrature. In the code we have chosen a very small $h\left(h=\frac{6-v}{2.000}\right)$ for the evaluation of the integrals in $f(u)$, and for the evaluation of $s_{j}, h=\frac{12}{100}$ since the error is of greater order $0\left(h^{4}\right)$.

Two Product Case - Example I

$$
\begin{aligned}
\text { Expected Prices }\left(\bar{p}_{i}\right) & \text { Standard Deviations }\left(\sigma_{i}\right) \\
\bar{p}_{1}=4.2426407 & \sigma_{1}=3.0 \\
\bar{p}_{2}=7.0710678 & \sigma_{2}=4.0
\end{aligned}
$$$$
\text { Market Shares (S }{ }_{i} \text { L (approximated) }
$$$$
S_{1}=0.7141959331
$$$$
S_{2}=0.0000000000
$$ 
From (3.11a) and (4.10) we can obtain the following analytical expression for $s_{1}$, where $\bar{p}_{21}=p_{2}-p_{1}, \sigma_{12}=\sqrt{\sigma_{1}^{2}+\sigma_{1}^{2}}$ :

$$
S_{1}=Q\left(\frac{\bar{p}_{21}}{\sigma_{12}}\right)=\frac{1}{2}+\frac{1}{2} \operatorname{erf}\left(\frac{\bar{p}_{21}}{\sqrt{2} \sigma_{12}}\right) \text {. }
$$

Finally we evaluate $S_{1}$ by using the tables of erf(u) given in Abramowitz et a $1 .,^{16}$ p. 310 , and compare:

$$
S_{1}=0.7141962 \text { (analytic). }
$$

Several other examples are given below for different values of $\bar{p}_{j}, \sigma_{i}$. This provides information on the sensitivlly ur market. shares to variations in expected prices and/or standard deviations (variability).

Two Product Case - Example 2

$$
\begin{array}{ll}
\text { Expected Prices }\left(\bar{p}_{i}\right) & \text { Standard Deviations }\left(\sigma_{i}\right) \\
\bar{p}_{1}=1.5000000 & \sigma_{1}=1.00 \\
\bar{p}_{2}=2.5000000 & \sigma_{2}=1.25
\end{array}
$$

Market Shares $\left(S_{i}\right)$

$$
\begin{aligned}
& S_{1}=0.7339141395 \\
& S_{2}=0.2660856802
\end{aligned}
$$


Two Product Case - Example 3

$$
\begin{aligned}
\text { Expected Prices }\left(\bar{p}_{j}\right) & \text { Standard Deviations }\left(\sigma_{i}\right) \\
\bar{p}_{1}=1.5000000 & \sigma_{1}=0.50 \\
\bar{p}_{2}=2.5000000 & \sigma_{2}=1.25
\end{aligned}
$$

$$
\begin{aligned}
& \frac{\text { Market Shares }\left(S_{i}\right)}{S_{1}=0.7711926108} \\
& S_{2}=0.2288070444
\end{aligned}
$$

Two Product Case - Example 4

$$
\begin{array}{cc}
\text { Expected Prices } \left.\bar{p}_{i}\right) & \text { Standard Deviations }(\sigma) \\
\hline \bar{p}_{1}=1.5000000 & \sigma_{1}=1.00 \\
\bar{p}_{2}=2.5000000 & \sigma_{2}=1.00 \\
\text { Market Shares }\left(S_{i}\right) & \\
S_{1}=0.7602497116 \\
S_{2}=0.2397501717
\end{array}
$$

Three Product Case - Example 5

$$
\begin{array}{ll}
\text { Expected Prices }\left(\bar{p}_{i}\right) & \text { Standard Deviations }\left(\sigma_{i}\right) \\
\bar{p}_{1}=1.5000000 & \sigma_{1}=1.00 \\
\bar{p}_{2}=2.0000000 & \sigma_{2}=0.50 \\
\bar{p}_{3}=2.5000000 & \sigma_{3}=1.00
\end{array}
$$

$$
\begin{aligned}
& \frac{\text { Market Shares }\left(S_{i}\right)}{S_{1}=0.5935275848} \\
& S_{2}=0.2458337014 \\
& S_{3}=0.1606383466
\end{aligned}
$$




\begin{tabular}{ll} 
Expected Prices $\left(\bar{p}_{i}\right)$ & Standard Deviations $\left(\sigma_{i}\right)$ \\
\hline $\bar{p}_{1}=1.000000$ & $\sigma_{1}=0.50$ \\
$\bar{p}_{2}=2.000000$ & $\sigma_{2}=1.50$ \\
$\bar{p}_{3}=3.000000$ & $\sigma_{3}=2.00$ \\
$\bar{p}_{4}=4.000000$ & $\sigma_{4}=3.00$ \\
$\bar{p}_{5}=5.000000$ & $\sigma_{5}=4.00$ \\
$\bar{p}_{6}=6.000000$ & $\sigma_{6}=2.00$ \\
$\bar{p}_{7}=8.000000$ & $\sigma_{7}=5.00$ \\
$\bar{p}_{8}=9.000000$ & $\sigma_{8}=7.00$ \\
$\bar{p}_{9}=10.000000$ & $\sigma_{9}=5.00$ \\
$\bar{p}_{10}=15.000000$ & $\sigma_{10}=2.00$
\end{tabular}

Market Shares $(S,)^{2}$

$$
\begin{aligned}
& s_{1}=0.34725 \\
& s_{2}=0.15300 \\
& s_{3}=0.09499 \\
& s_{4}=0.10600 \\
& s_{5}=0.11476 \\
& s_{6}=0.00070 \\
& s_{7}=0.05727 \\
& s_{8}=0.10174 \\
& s_{9}=0.02404 \\
& s_{10}=0.00000
\end{aligned}
$$




\section{AVERAGE PRICES}

The present practice in the DFI model is to pass a price to the consumer.which is the average of market share prices. For $\mathrm{N}=2$ we have

$$
\overline{\mathrm{p}}=\mathrm{S}_{1} \overline{\mathrm{p}}_{1}+\mathrm{S}_{2} \overline{\mathrm{p}}_{2} \text {. }
$$

Notice that this implies the average price faced by consumers is greater than the minimum of the average prices $\overline{\mathrm{p}}_{1}, \overline{\mathrm{p}}_{2}$. We would expect the actual average price to be smaller than the minimum of $\left(\bar{p}_{1}, \bar{p}_{2}, \ldots, \bar{p}_{N}\right)$ by the "rational consumer behavior" assumption. The rational consumer behavior assumption would require the average price to be computed as

$$
\bar{p}=\sum_{i \neq j} E\left(x_{i} \mid x_{i} \leq x_{j}\right)
$$

For the case of two products $(N=2)(5.2)$ becomes

$$
\begin{aligned}
\bar{p} & =E\left(x_{1} \mid x_{1} \leq x_{2}\right)+E\left(x_{2} \mid x_{2} \leq x_{1}\right) \\
& =\int_{-\infty}^{\infty} \int_{x_{1}}^{\infty} x_{1} f\left(x_{1}, x_{2}\right) d x_{1} d x_{2}+\int_{-\infty}^{\infty} \int_{x_{2}}^{\infty} x_{2} f\left(x_{1}, x_{2}\right) d x_{1} d x_{2} .
\end{aligned}
$$

In the general case of $\mathrm{N}$ products we have

$$
\bar{p}=\sum_{i=1}^{N} \int_{-\infty}^{\infty} \int_{x_{i}}^{w} \ldots \int_{x_{i}}^{\infty} x_{i} f\left(x_{1}, x_{2}, \ldots, x_{N}\right) d x_{1} d x_{2} \ldots d x_{N} \text {. }
$$

To gain some insight into the potential price differences resulting from equalion (5.2) versus equation (5.1) we will examine the case of two products for a normal distribution in some detail. In the case of $N$ products we will 
develop a numerical code similar to the one for market shares.

Let us introduce relation (3.4) in

$$
\begin{aligned}
& E\left(x_{1} \mid x_{1} \leq x_{2}\right)=\int_{-\infty}^{\infty} \int_{x_{1}}^{\infty} x_{1} f\left(x_{1}\right) f\left(x_{2}\right) d x_{1} d x_{2} \\
& =\frac{1}{2 \eta} \int_{-\infty}^{\infty} F_{1}\left(t_{1}\right)\left(\int_{-\infty}^{\infty} x_{1} \exp \left(-i x_{1} t_{1}\right) d x_{1}\right) d t_{1} \int_{-\infty}^{\infty} F_{2}\left(t_{2}\right) \\
& \text { - }\left(\int_{x_{1}}^{\infty} \exp \left(-i x_{2} t_{2}\right) d x_{2}\right) d t_{2} \\
& =\frac{1}{2 \pi} \int_{-\infty}^{m} \cdot F_{1}\left(t_{1}\right)\left(\int_{-\infty}^{\infty} x_{1} \exp \left(-i x_{1} t_{1}\right) d x_{1}\right) d t_{1} \int_{-\infty}^{m} F_{2}\left(t_{2}\right) \\
& \text { - } \exp \left(-i t_{2} x_{1}\right)\left\{\pi \delta\left(t_{2}\right)-\frac{i}{t_{2}}\right\} d t_{2} \\
& =\frac{1}{2 \pi}\left\{\int_{-\infty}^{\infty} F_{1}\left(t_{1}\right)\left(\int_{-\infty}^{\infty} x_{1} \exp \left(-i x_{1} t_{1}\right) d x_{1}\right) d t_{1}\right. \\
& \text { - } \pi F_{2}(0)-i \int_{-\infty}^{\infty} F_{1}\left(t_{1}\right)\left(\int_{-\infty}^{\infty} x_{1} \exp \left(-i x_{1} t_{1}\right) d x_{1}\right) d t \\
& \left.\cdot \int_{-\infty}^{\infty} \frac{F_{2}\left(t_{2}\right) \exp \left(-i t_{2} x_{1}\right)}{t_{2}} d t_{2}\right\} \\
& =\frac{1}{2 \pi}\left\{\int_{-\infty}^{\infty} F_{1}\left(t_{1}\right) \frac{d}{d t_{1}}\left(i \int_{-\infty}^{\infty} \exp \left(-i x_{1} t_{1}\right) d x_{1}\right) d t_{1}\right. \\
& \text { - } \left.\pi F_{2}(0)-i \int_{-\infty}^{\infty} F_{1}\left(t_{1}\right) d t_{1} \int_{-\infty}^{\infty} F_{2}\left(l_{2}\right) \int_{-\infty}^{\infty} \ddot{x}_{1} \exp \left[i\left(t_{1}+t_{n_{2}}\right) x_{1}\right] d x_{1}\right] \\
& =\frac{1}{2 \pi}\left\{i \pi F_{2}(0) \int_{-\infty}^{\infty} F_{1}\left(t_{1}\right) 2 \pi \delta^{\prime}\left(t_{1}\right) d t_{1}-i \cdot i \int_{-\infty}^{\infty} F_{1}\left(t_{1}\right) d t_{1}\right. \\
& \cdot \int_{-\infty}^{\infty} \frac{F_{2}\left(t_{2}\right)}{t_{2}} \cdot 2 \pi \delta^{\prime}\left(t_{1}+l_{2}\right) d t_{2}
\end{aligned}
$$

- $28-$ 


$$
\begin{aligned}
&=\frac{1}{2 \pi}\left\{2 i \pi^{2} F_{2}(0):(-1) F_{j}^{\prime}(0)+2 \pi \int_{-\infty}^{\infty} F_{1}\left(t_{1}\right)\right. \\
&\left.\cdot\left(\frac{F_{2}^{\prime}\left(-t_{1}\right)}{t_{1}}+\frac{F_{2}\left(-t_{1}\right)}{t_{1}{ }^{2}}\right) d t_{1}\right\} \\
&=\frac{1}{2 \pi}\left\{-2 \pi^{2} i F_{2}(0) F_{1}^{\prime}(0)+2 \pi \int_{-\infty}^{\infty} \frac{F_{1}\left(t_{1}\right) F_{2}^{\prime}\left(-t_{1}\right)}{t_{1}} d t_{1}\right. \\
&+2 \pi \int_{-\infty}^{\infty} \frac{F_{1}\left(t_{1}\right) F_{2}\left(-t_{1}\right)}{t_{1}{ }^{2}} \cdot d t \\
&=-\pi i F_{2}(0) F_{1}^{\prime}(0)+\int_{-\infty}^{\infty} \frac{F_{1}\left(t_{1}\right) F_{2}^{\prime}\left(-t_{1}\right)}{t_{1}} d t_{1}-\left.\frac{F_{1}\left(t_{1}\right) F_{2}\left(-t_{1}\right)}{t_{1}}\right|_{-\infty} ^{\infty} \\
&+\int_{-\infty}^{\infty} \frac{1}{t_{1}}\left\{F_{1}^{\prime}\left(t_{1}\right) F_{2}\left(-t_{1}\right)-F_{1}\left(t_{1}\right) F_{2}^{\prime}\left(-t_{1}\right)\right\} d t_{1} \cdot
\end{aligned}
$$

Final $11 y$

$$
E\left[x_{1} \mid x_{1} \leq x_{2}\right]=-\pi i F_{2}(0) F_{1}^{\prime}(0)+\int_{-\infty}^{\infty} \frac{F_{1}^{\prime}\left(t_{1}\right) F_{2}\left(-t_{1}\right)}{t_{1}} d t_{1},
$$

and similarly

$$
E\left[x_{2} \mid x_{2} \leq x_{1}\right]=-i \pi F_{2}^{\prime}(0) F_{1}(0)+\int_{-\infty}^{\infty} \frac{F_{2}^{\prime}(t) F_{1}(-t)}{t} d t \text {. }
$$

If $f_{j}(t)$ is normal then

$$
F_{j}(0)=\frac{1}{\sqrt{2} \pi}, \quad F_{j}^{\prime}(0)=\frac{i}{\sqrt{2} \pi} \bar{p}_{j}, \bar{p}_{j}=\int_{-\infty}^{\infty} x f_{j}(x) d x
$$

and from (4.2),

$$
F_{j}^{\prime}(t)=\frac{1}{\sqrt{2} \pi} \exp \left(i t \bar{p}_{j}\right) \exp \left(-\frac{\sigma_{j}^{2} t^{2}}{2}\right)\left\{i \bar{p}_{j}-\sigma_{j}^{2} t\right\} \text {. }
$$

Substituting the last equation in (5.6) we obtain

$-29-$ 


$$
\begin{aligned}
E\left[x_{1} \mid x_{1} \leq x_{2}\right] & =\frac{\bar{p}_{1}}{2}+\frac{1}{2 \pi} \int_{-\infty}^{\infty} \frac{\exp \left(-i t \bar{p}_{21}-\frac{\sigma_{12}^{2} t^{2}}{2}\right)}{t}\left\{i \bar{p}_{1}-\sigma_{1}^{2} t\right\} d t \\
& =\frac{\bar{p}_{1}}{2}+\frac{i \bar{p}_{1}}{2 \pi} \int_{-\infty}^{\infty} \frac{\exp \left(-i t \bar{p}_{21}-\frac{\sigma_{12}^{2} t^{2}}{2}\right)}{t} d t \\
& =\bar{p}_{1}\left\{\frac{1}{2}+\frac{i}{2 \pi} \int_{-\infty}^{\infty} \frac{\exp \left(-i t \bar{p}_{21}-\frac{\sigma_{12}^{2} t^{2}}{2}\right)}{t} d t\right\} \\
& -\frac{\sigma_{1}^{2}}{i_{2 \pi}^{2}} 2^{-\frac{1}{2}\left(\frac{\sigma_{12}}{\sqrt{2}}\right)-1} \exp \left(-i t \bar{p}_{21}\right) \exp \left(-\frac{\sigma_{12}^{2} t^{2}}{2}\right) d t \\
& \left.=\bar{p}_{1} S_{1}-\frac{p_{12}^{2}}{\sigma_{12}^{2}}\right)
\end{aligned}
$$

where we have used the known result,

$$
S_{1}=\frac{1}{2}+\frac{i}{2 \pi} \int_{-\infty}^{\infty} \frac{\exp \left(-i t \bar{p}_{21}-\frac{\sigma_{22}^{2} t^{2}}{2}\right)}{t} \text {. }
$$

If we use the symmetry of the problem we find that

$$
\begin{aligned}
& E\left[x_{1} \mid x_{1} \leq x_{2}\right]=\bar{p}_{1} s_{1}-\frac{\sigma_{1}^{2}}{\sqrt{2 \pi} \sigma_{12}} \exp \left(-\frac{\bar{p}_{12}^{2}}{2 \sigma_{12}^{2}}\right), \\
& E\left[x_{2} \mid x_{2} \leq x_{1}\right]=\bar{p}_{2} s_{2}-\frac{\sigma_{2}^{2}}{\sqrt{2 \pi} \sigma_{12}} \exp \left(-\frac{\bar{p}_{21}^{2}}{2 \sigma_{12}^{2}}\right) .
\end{aligned}
$$

If we add the equations (5.8) we get,

$$
\ddot{p}=\bar{p}_{1} S_{1}+\bar{p}_{2} s_{2}-\frac{\sigma_{12}}{\sqrt{2 \pi}} \exp \left(-\frac{\bar{p}_{3}^{2}{ }_{2}}{2 \sigma_{12}^{2}}\right) \text {. }
$$

The implication for the algorithm is that the perceived consumer price average is less than $\bar{p}_{1} S_{1}+\bar{p}_{2} S_{2}$ by an amount proportional to the combined uncertainty, $\sigma_{12}$, and 
functionally dependent on the normal distribution. Consequently, the final prices found by the model are likely to be overestimates of the market clearing prices. This then implies that the market is not in equilibrium.

For the general case of $N$ products we use the same direct numerical method for the evaluation of the average prices described in section IV. The only difference in the numerical approximation is that in (4.22) the function $\exp \left(-u^{2}\right) f(u)$ is now replaced by $\left(u \sigma_{i} \sqrt{2}+p_{i}\right) \exp \left(-u^{2}\right) f(u)$. The code developed here finds the market shares and average prices for any arbitrary N. A numerical example below shows the agreement of the numerical method and the analytical one for average prices.

Two Product Case - Example 7

$$
\begin{aligned}
\text { Expected Prices }\left(\bar{p}_{i}\right) & \text { Standard Deviations }\left(\sigma_{i}\right) \\
\bar{p}_{1}=4.24264070 & \sigma_{1}=3.00 \\
\bar{p}_{2}=7.07106780 & \sigma_{2}=4.00
\end{aligned}
$$

Market Shares $\left(S_{i}\right)$

$$
\begin{aligned}
& S_{1}=0.7141959334 \\
& S_{2}=0.2858038765
\end{aligned}
$$

Approximate average price $=3.3512352$, $\underline{\text { Exact }}=p_{1} S_{1}+p_{2} S_{2}-\frac{\sigma_{12}}{\sqrt{2 \pi}} \exp \left(-\frac{p_{12}^{2}}{2 \sigma_{12}^{2}}\right)=3.3512337$. 
VI. SENSITIVITY OF MARKET SHARES TO THE FORM OF PROBABILITY FUNCTION

One of the important issues which arises when considering probabilistic interpretations of market shares, and indeed any model, is the sensitivity of the model to the type of probability distribution selected. Results that are not extremely sensitive to the choice of p.d.f. imply that less care (or empirical work) is necded to calibrate and use the model.

Sellsilive results imply that great care must be taken to collect and analyze the needed data before the model can be usefully applied. We examine the sensitivity issue by deriving the market share formulations for uniform and gamma distributions. The uniform distribution was selected for its simplicity, and the gamma distribution was selected for its more realistic representation of market behavior.

The implications are intuitively appealing. The results are moderately sensitive to the choice of distribution. There are substantial differences implied by the choice of uniform distributions as contrasted to either the normal or gamma distributions. However, the market shares results for gamma versus the normal distribution do not appear to be substantially different for the cases studied. The details follow below.

\section{Uniform Distribution}

Consider the following probability density functions (p.d.f.'s) (Figure 2). 


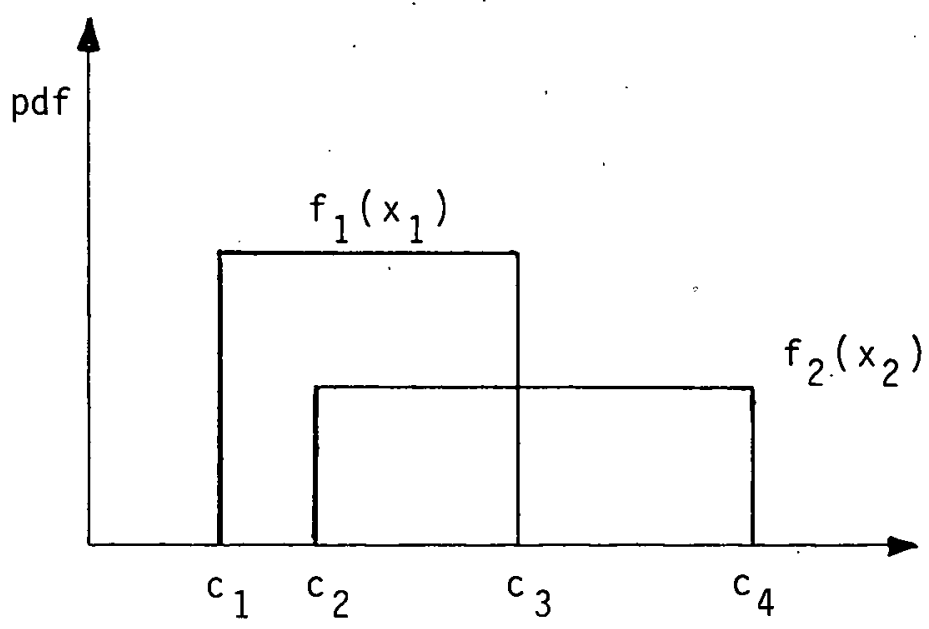

Figure 2. Two Product Case; Uniform Distributions

That is,

$$
\begin{aligned}
& f_{1}\left(x_{1}\right)= \begin{cases}\frac{1}{c_{3}-c_{1}}, & c_{1} \leq x_{1} \leq c_{3} \\
0, & \text { elsewhere }\end{cases} \\
& f_{2}\left(x_{2}\right)= \begin{cases}\frac{1}{c_{4}-c_{2}}, & c_{2} \leq x_{2} \leq c_{4} \\
0, & \text { elsewhere. }\end{cases}
\end{aligned}
$$

The mean $\bar{p}_{1}$ and standard deviation $\sigma_{1}$ for the p.d.f. $f_{1}$ defined in (6.1) are given by

$$
\begin{aligned}
& \bar{p}_{1}=\frac{c_{3}+c_{1}}{2}, \\
& \sigma_{1}^{2}=\frac{\left(c_{3}-c_{1}\right)^{2}}{12} .
\end{aligned}
$$

We repeat the definition of market shares for two products (formula 3.1 ) below.

$$
s_{i}=\int_{-\infty}^{\infty} f_{1}\left(x_{1}\right) d x_{1} \int_{x_{1}}^{\infty} f_{2}\left(x_{2}\right) d x_{2} .
$$

Substituting the uniform distribution. (6.1) in (6.2) we get 


$$
\begin{aligned}
s_{1} & =\frac{1}{c_{3}-c_{1}} \int_{c_{1}}^{c_{3}} d x_{1} \int_{x_{1}}^{c_{4}} f_{2}\left(x_{2}\right) d x_{2} \quad\left(x_{1} \leq x_{2}\right) . \\
& =\frac{1}{c_{3}-c_{1}}\left\{\int_{c_{1}}^{c_{2}} d x_{1} \int_{c_{2}}^{c_{4}} \frac{1}{c_{4}-c_{2}} d x_{2}+\int_{c_{2}}^{c_{3}} d x_{1} \int_{x_{1}}^{c_{4}} \frac{1}{c_{4}-c_{2}} d x_{2}\right\} .
\end{aligned}
$$

After integration this becomes

$$
S_{1}=\frac{1}{\left(c_{3}-c_{1}\right)\left(c_{4}-c_{2}\right)}\left(\left(c_{2}-c_{1}\right)\left(c_{4}-c_{2}\right)-\frac{\left(c_{4}-c_{3}\right)^{2}}{2}+\frac{\left(c_{4}-c_{2}\right)^{2}}{2}\right]
$$

which can be rewritten as

$$
s_{1}=1-\frac{\left(\frac{c_{3}}{2}-\frac{c_{2}}{4} \cdot\right)_{1}^{2}}{\sigma_{1} \cdot \sigma_{2}} \text {. }
$$

Similarly, we find that

$$
s_{2}=\frac{1}{\left(c_{4}-c_{2}\right)\left(c_{3}-c_{1}\right)} \cdot \frac{\left(c_{3}-c_{2}\right)^{2}}{2}=\frac{\left(c_{3}-c_{2}\right)^{2}}{24 \cdot \sigma_{1} \cdot \sigma_{2}} \text {. }
$$

Two Product Case - Example 8.

$$
\begin{array}{cc}
\text { Expected Prices }\left(\bar{p}_{i}\right) & \frac{\text { Standard Deviations }\left(\sigma_{i}\right)}{\bar{p}_{1}=1.500} \\
\bar{p}_{p_{2}}=2.500 & \sigma_{1}=1.000 \\
\sigma_{2}=1.000
\end{array}
$$

$$
\begin{aligned}
& \frac{\text { Market Shares }\left(S_{i}\right)}{S_{1}}=0.7602 \text { (Normal distribution) } \\
& S_{2}=0.2398
\end{aligned}
$$

If we consider uniform distributions with the same expected values ond standard deviatinns, we can find $c_{1}, c_{2}, c_{3}, c_{1}$ in (6.3) from the formulas

$$
\bar{p}_{1}=\frac{c_{1}+c_{3}}{2}, \quad \sigma_{1}^{2}=\frac{\left(c_{3}-c_{1}\right)^{2}}{12}, \ldots,
$$




$$
\begin{array}{ll}
c_{1}=-0.2321 & c_{2}=0.7679 \\
c_{3}=3.2321 & c_{4}=4.2321
\end{array}
$$

Finally the market shares for the uniform distributions can be found from $(6.3),(6.4)$, and $(6.5)$ :

$$
\begin{aligned}
& s_{1}=0.8970 \\
& s_{2}=0.1031
\end{aligned}
$$

\section{Gamma Distribution}

The gamma distribution p.d.f. is defined by

$$
f\left(x_{i}\right)= \begin{cases}\frac{\alpha_{i}}{\Gamma\left(r_{i}\right)}\left(\alpha_{i} x_{i}\right)^{r_{i}-1} \exp \left(-\alpha_{i} x_{i}\right), & x_{i}>0 \\ 0, & \text { elsewhere (6.6) }\end{cases}
$$

where the mean of such a distribution is $\bar{p}_{i}=\frac{r_{i}}{\alpha_{i}}$ and the variance is $\sigma_{i}^{2}=\frac{r_{i}}{\alpha_{i}{ }^{2}}$.

Notice that the p.d.f. is positive for $x_{i}>0$ and zero elsewhere, which implies that "free goods" are precluded. The market share of product one is given by.

$$
s_{1}=\int_{-\infty}^{\infty} f_{1}\left(x_{1}\right) d x_{1} \int_{x_{1}}^{\infty} f_{2}\left(x_{2}\right) d x_{2} .
$$

Notice that

$$
\Gamma(p)=\int_{0}^{\infty} x^{p-1} \exp (-x) d x .
$$

If we assume the $r_{i}$ are integers then $\Gamma\left(r_{i}\right)=\left(r_{i}-1\right)$ !. Substituting (6.6) into (6.7) (see Meyer, ${ }^{18} \mathrm{p}$. 195) we obtain 


$$
\begin{aligned}
S_{1} & =\frac{\alpha_{1}}{\Gamma\left(r_{1}\right)} \int_{0}^{\infty}\left(\alpha_{1} x_{1}\right)^{r_{1}-1} \exp \left(-\alpha_{1} x_{1}\right) d x_{1} \int_{x_{1}}^{\infty} \alpha_{2} \frac{\left(\alpha_{2} x_{2}\right)^{r_{2}-1}}{\Gamma\left(r_{2}\right)} \exp \left(-\alpha_{2} x_{2}\right) d x_{2} \\
& =\frac{\alpha_{1}}{\Gamma\left(r_{1}\right)} \int_{0}^{\infty}\left(\alpha_{1} x_{1}\right)^{r_{1}-1} \exp \left(-\alpha_{1} x_{1}\right) d x_{1} \sum_{k=0}^{r_{2}-1} \exp \left(-\alpha_{2} x_{1}\right)\left(\alpha_{2} x_{1}\right)^{k} / k ! \\
& =\frac{\alpha_{1}}{\Gamma\left(r_{1}\right)} \sum_{k=0}^{r_{2}-1} \frac{\alpha_{1}^{r_{1}-1} \alpha_{2}}{k !} \int_{0}^{\infty} x_{1}^{r_{1}-1+k} \exp \left[-\left(\alpha_{1}+\alpha_{2}\right) x_{1}\right] d x_{1} .
\end{aligned}
$$

If we let $\left(\alpha_{1}+\alpha_{2}\right) x_{1}=u$ then

$$
\begin{aligned}
s_{1} & =\frac{\alpha_{1}}{\Gamma\left(r_{1}\right)} \sum_{k=0}^{r_{2}-1} \frac{\alpha_{1}^{r_{1}-1} r_{r_{3}} k}{k !\left(\alpha_{1}+\alpha_{2}\right)^{r_{1}+k}} \int_{0}^{\infty} u^{r_{1}-1+k_{\text {exp }}(=u) d u} \\
& =\frac{\alpha_{1}}{\Gamma\left(r_{1}\right)} \sum_{k=0}^{r_{2}} \frac{\alpha_{1}-1 \alpha_{2}^{k}}{k !\left(\alpha_{1}+\alpha_{2}\right)^{r_{1}+k}} \Gamma\left(r_{1}+k\right) .
\end{aligned}
$$

Weibull Distribution

The market allocation formulation within the Decision Focus, Inc. (DFI) energy, equilibrium mode ${ }^{19}$ has occasionally shown some unusual behavior and has prompted interest in deriving the probabilistic formulation which is equivalent to the DFI formulation. Static market shares in the DFI model are defined by the relation

$$
s_{i}=\frac{f_{i} p_{j}^{-\gamma}}{\sum_{j=1}^{n} f_{j} p_{j}^{-\gamma}}
$$

where the $p_{i}$ can be viewed as the mean prices of some price distribution, the $f_{i}$ are weighting factors which indicate any inherent bias by consumers under equal price assumptions, and $\gamma$ is the price sensitivity parameter. 
D. B. Reister and B. Phillips have independently shown in unpublished technical memoranda that the DFI static market shares formulation is equivalent to assuming that prices are independent random variables from weibull distributions of the form

$$
f(x)=\alpha \gamma x^{\gamma-1} \exp \left(-\alpha x^{\gamma}\right)
$$

The mean price $\bar{p}$ and variance, $\sigma^{2}$, are defined by

$$
\begin{aligned}
\overline{\mathrm{p}} & =\alpha^{-1 / \gamma} \Gamma(1+1 / \gamma), \\
\sigma^{2} & =\alpha^{-2 / \gamma}\left[\Gamma(1+2 / \gamma)-\Gamma^{2}(1+1 / \gamma)\right] .
\end{aligned}
$$

Notice that $\Gamma(p)$ is positive for $x$ positive, and the maximum value of $\Gamma(p)$ is attained in the interval $p \in(1,2)$. Reister and Phillips have shown that the static market shares, using the Weibull distribution with identical values of $\gamma$ and arbitrary $\alpha_{i}$, reduce to

$$
s_{i}=\frac{\alpha_{i}}{\sum_{j=1}^{n} \alpha_{j}}=\frac{p_{i}^{-\gamma}}{\sum_{j \equiv 1}^{n} p_{j}^{-\gamma}} .
$$

We examine the behavior of the Weibull distribution and relate it to the implications for market shares.

For large values of $\gamma$ the quantities $(1+1 / \gamma)$ and $(1+2 / \gamma)$ approach one and $\sigma^{2}$ approaches zero while the mean price $\bar{p}$ approaches one. For example, for values of $\gamma \geq 20$ and $0.1 \leq \alpha \leq 100$ 


$$
\begin{aligned}
& 0.77 \leq \bar{p} \leq 1.09 \text { and } \\
& 0.0023 \leq \sigma^{2} \leq 0.0045 .
\end{aligned}
$$

The current parameter values for $\gamma$ used in the DFI model belong to the range $(10,40)$. This implies that fuel prices from

all suppliers tend to $\$ 1.00$ with very small variability. See Figure 3 , below:

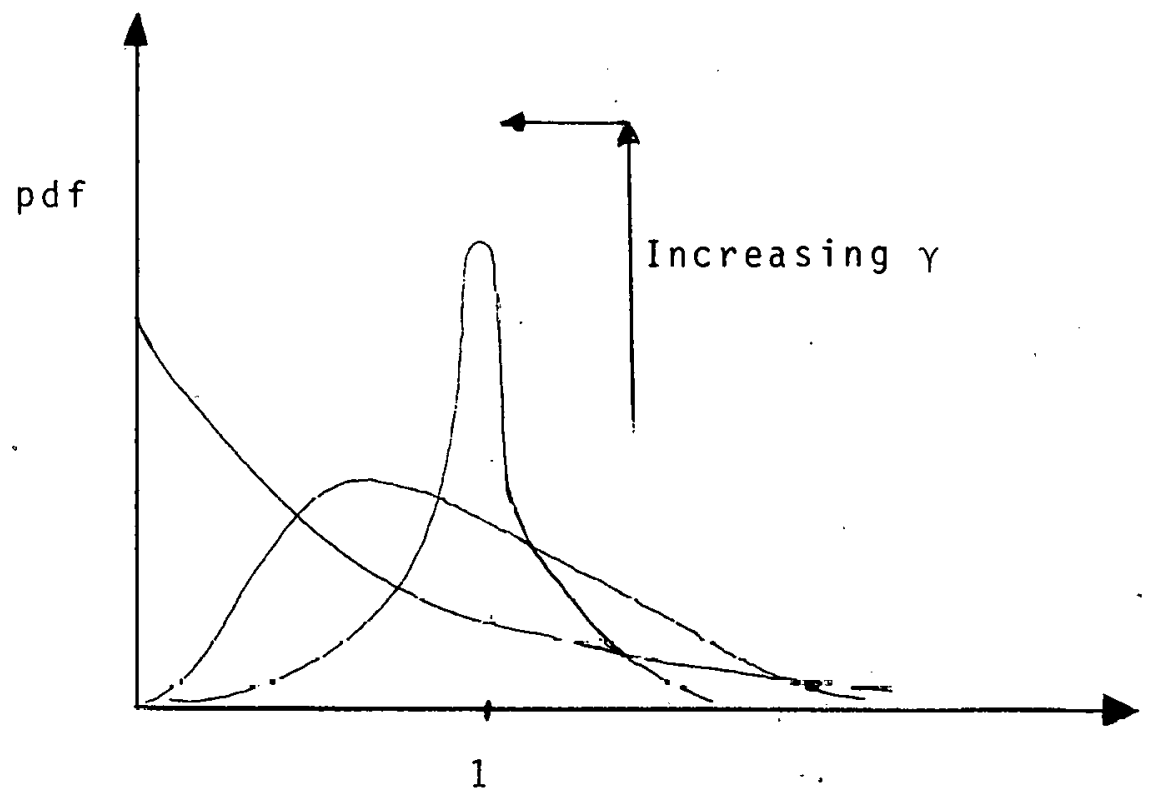

Figure 3. The Weibull Density Function

In view of these observations, it is not surprising when small changes in mean prices provide large changes in static market shares for large values of $\gamma$. As an example consider the two-product case below. Notice that a slight shift of the mean 
price would drastically alter the market shares due to the narrow cost band implied by the price distributions. See Figure 4.

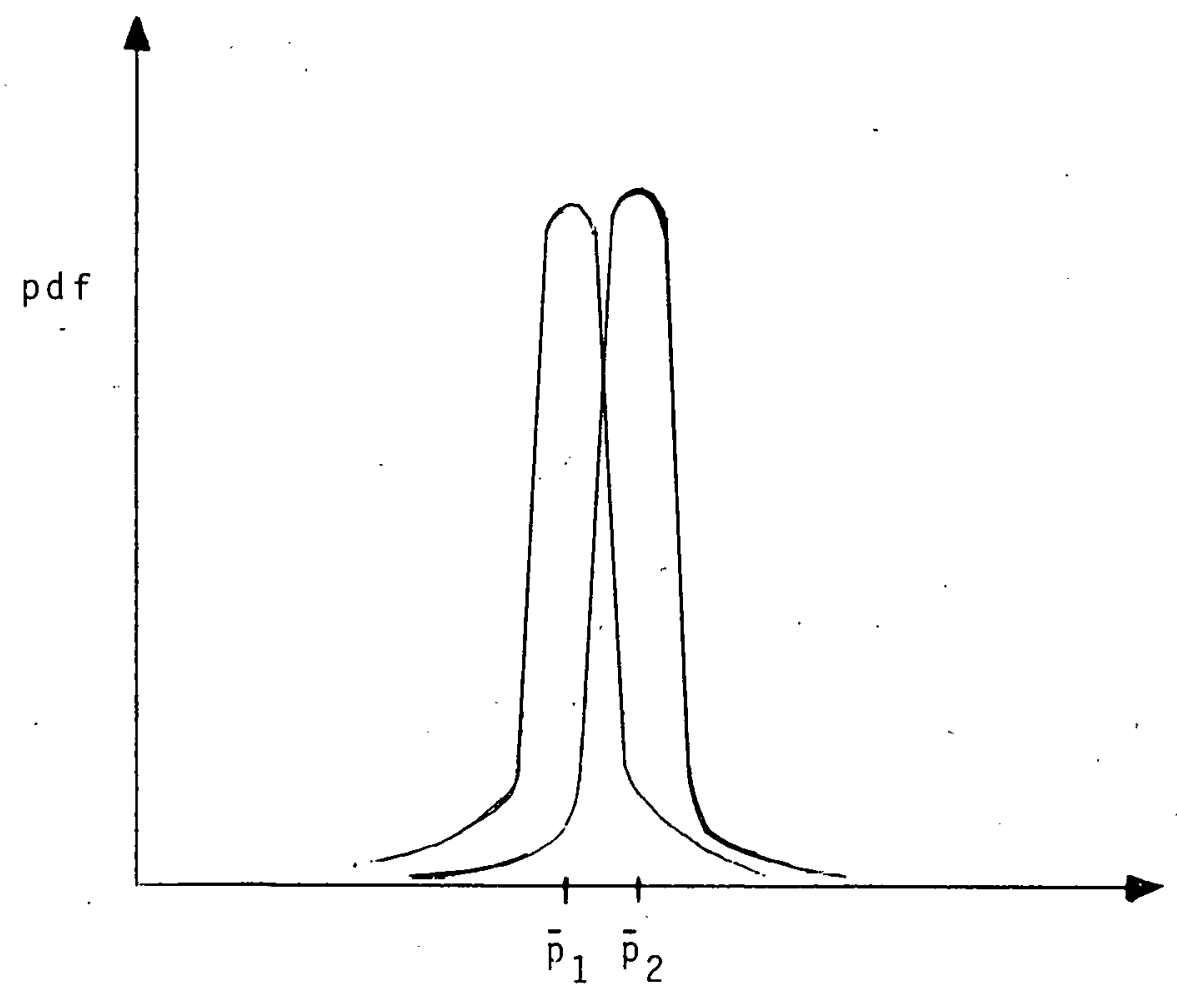

Figure 4. Sensitivity of Market Shares for the Weibull Distribution with Large $\gamma$.

Additional empirical study will be required to determine the most appropriate frequency distribution and resulting market allocation formulation in the DFI model. It appears, oil serisitivity grounds, that the Weibull distribution with large $\gamma$ is inappropriate for deriving the market allocation formula. 


\section{Additional Examples}

Two Product Case - Example 9

Assume that $r_{1}=4.0, r_{2}=2.0$ and $\alpha_{1}=\alpha_{2}=1$, then

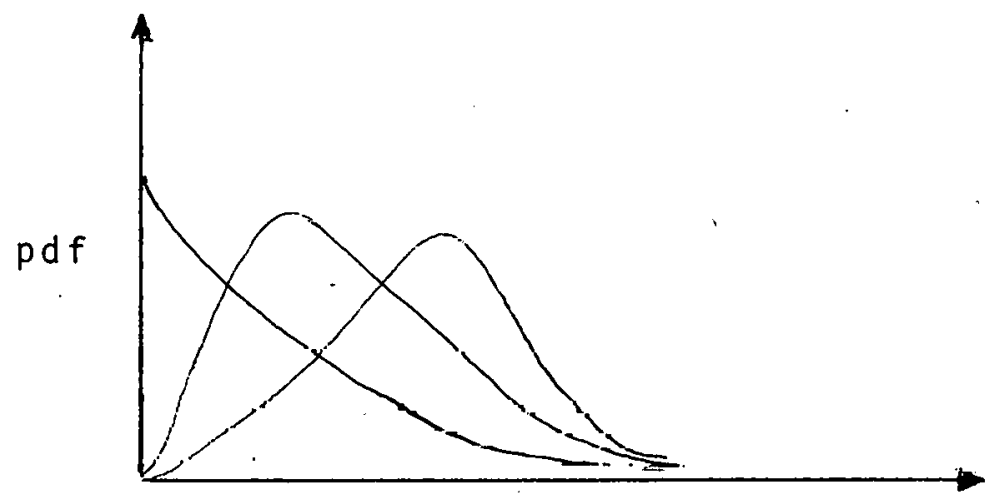

Figure 5. Behavior of the Gamma Distribution.

From (6.6) the market share for product one is

$$
\begin{aligned}
S_{1} & =\frac{\alpha_{1}}{\Gamma\left(r_{1}\right)}\left\{\frac{\alpha_{1}^{r_{1}-1} \alpha_{2}^{0}}{\left(\alpha_{1}+\alpha_{2}\right)^{r_{1}}} \Gamma\left(r_{1}\right)+\frac{\alpha_{1}^{r_{1}-1} \alpha_{2}}{\left(\alpha_{1}+\alpha_{2}\right)^{r_{1}+1}} \Gamma\left(r_{1}+1\right)\right\} \\
& =\alpha_{1}\left\{\frac{\alpha_{1}^{r_{1}-1}}{\left(\alpha_{1}+\alpha_{2}\right)^{r_{1}}}+\frac{\alpha_{1}^{r_{1}-1} \alpha_{2}}{\left(\alpha_{1}+\alpha_{2}\right)^{r_{1}+1}} r_{1}\right\} .
\end{aligned}
$$

Hence $S_{1}=0.19$ and $S_{2}=0.81$. Using the formulas for the mean prices $\left(\bar{p}_{i}\right)$ and variances $\left(\sigma_{i}{ }^{2}\right), \bar{p}_{i}=\frac{r_{i}}{\alpha_{2}}, \sigma_{i}{ }^{2}=\frac{r_{i^{2}}}{\alpha_{i}}$, we find

$$
\begin{aligned}
& \frac{\text { Expected Prices }\left(\bar{p}_{j}\right)}{\bar{p}_{1}=4.0} \\
& \text { Standard Deviations }\left(\sigma_{i}\right) \\
& \bar{p}_{2}=2.0 \\
& \begin{array}{l}
\sigma_{1}=2.0 \\
\sigma_{2}=\sqrt{2}
\end{array}
\end{aligned}
$$




$$
\begin{aligned}
& \frac{\text { Market Shares }\left(\dot{S}_{j}\right)}{S_{1}=0.21} \text { (Normal distribution) } \\
& S_{2}=0.79 \\
& \text { Market Shares }\left(S_{j}\right) \\
& S_{1}=0.11 \\
& S_{2}=0.89
\end{aligned}
$$

Two Product Case - Example 10

We assume that $r_{1}=3, r_{2}=2, \alpha_{i}=1$ then

$$
\begin{array}{cl}
\text { Expected Prices }\left(p_{i}\right) \\
\hline \bar{p}_{1}=3.0
\end{array} \quad \begin{aligned}
& \text { Standard Deviations }\left(\sigma_{i}\right) \\
& \bar{p}_{2}=2.0 \sigma_{1}=\sqrt{3} \\
& \sigma_{2}=\sqrt{2}
\end{aligned}
$$

The market share of product one for the gamma distribution is

$$
S_{1}=0.31 \text { (gamma) }
$$

while the normal gives

$$
S_{1}=0.33 \text { (normal). }
$$

Evidently the market shares estimates assuming either a normal price distribution or a gamma price distribution with relatively few degrees of freedom give very similar results. The uniform distribution can give somewhat different results than either normal or gamma distributions. For long-range planning the differences or errors implied may be insignificant. 


\section{REFERENCES}

1. A. P. Hurter Jr. and A. H. Rubenstein, Market Penetration by New Innovations: The Technological Literature, Elsevier North-Holland, Amsterdam, 1978.

2. R. Condap and A. Kydes, Modeling Market Penetration with Emphasis on the DFI Energy-Economy System, BNL 50999, 1979.

3. F. M. Bass, A new product growth model for consumer durables, Management Sci. 15, 215-27 (1969).

4. A. W. Blackman Jr., A mathematical model for trend furecasts, Tech. Forccasting soc. Change 3, 441-5? (197?).

5. A. W. Blackman Jr., An innovation index based on factor analysis, Ibid. 4 , 301-16 (1972).

6. A. W. Blackman Jr., The market dynamics of technological substitutions, Ibid. $\underline{6}, 41-63$ (1974).

7. E. Mansfield, Technical change and the rate of initiation, Econometrica 29,741-65 (1961).

8. J. C. Fisher and R. H. Pry, A simple substitution model for technological change, Tech. Forecasting Soc. Change 3 , $75-88(1971)$.

9. V. Peterka, Macrodynamics of Technological Change: Market Penetration by New Technologies, International Institute for Applied Systems Analysis, RR-77-22, 1977.

10. R. E. Bohn and J. S. Herod, Industrial Sector Technology Use (ISTUM): Industrial Energy Use in the United States, 1974-2000, Vol. 1, Preliminary Model Ducumentation, Book 2, 
Chap. IV to VII, Prepared for DOE by Energy and Environmental Analysis, Inc., Arlington, VA, 1978.

11. V. Peterka and F. Fleck, The Dynamics of Energy Systems and the Logistic Substitution Model, Vol. 2, International Institute for Applied Systems Analysis, July 1978.

12. R. V. Hogg and A. T. Craig, Introduction to Mathematical Statistics, 3rd ed., Macmillan, London, 1970.

13. I. H. Sneddon, The Use of Integral Transforms, McGraw-Hi11, New York, 1972.

14. F. G. Tricomi, Integral Equations, Interscience, New York, 1957.

15. A. Erdelyi, W. Magnus, F. Oberhettinger, and F. G. Tricomi, Tables of Integral Transforms, 2 Vol., McGraw-Hill, New York, 1954.

16. M. Abramowitz and I. A. Stegun, Handbook of Mathematical Functions, Dover, New York, 1968.

17. G. Dahlquist, A. Björck, and N. Anderson, Numerical Methods, Prentice-Hall, Englewood Cliffs, NJ, 1974.

18. P. L. Meyer, Introduction Probability and Statistical Applications, 2nd ed., Addison-Wesley, New York, 1970

19. R. J. Alder, E. G. Cazalet, S. M. Haas, R. A. Marshalla, D. M. Nesbitt, and R. L. Phillips, The DFI Energy-Economy Modeling System, Draft Rep., Decision Focus, Inc., Palo Alto, CA, 1978. 